\title{
On the Longest Edge of Gabriel Graphs in Wireless Ad Hoc Networks
}

\author{
Peng-Jun Wan and Chih-Wei Yi, Member, IEEE
}

\begin{abstract}
In wireless ad hoc networks, without fixed infrastructures, virtual backbones are constructed and maintained to efficiently operate such networks. The Gabriel graph (GG) is one of widely used geometric structures for topology control in wireless ad hoc networks. If all nodes have the same maximal transmission radii, the length of the longest edge of the GG is the critical transmission radius such that the GG can be constructed by localized and distributed algorithms using only 1-hop neighbor information. In this paper, we assume a wireless ad hoc network is represented by a Poisson point process with mean $n$ on a unit-area disk, and nodes have the same maximal transmission radii. We give three asymptotic results on the length of the longest edge of the GG. First, we show that the ratio of the length of the longest edge to $\sqrt{\frac{\ln n}{\pi n}}$ is asymptotically almost surely equal to 2 . Next, we show that for any $\xi$, the expected number of GG edges whose lengths are at least $2 \sqrt{\frac{\ln n+\xi}{\pi n}}$ is asymptotically equal to $2 e^{-\xi}$. This implies that $\xi \rightarrow \infty$ is an asymptotically almost sure sufficient condition for constructing the GG by 1-hop information. Last, we prove that the number of long edges is asymptotically Poisson with mean $2 e^{-\xi}$. Therefore, the probability of the event that the length of the longest edge is less than $2 \sqrt{\frac{\ln n+\xi}{\pi n}}$ is asymptotically equal to $\exp \left(-2 e^{-\xi}\right)$.
\end{abstract}

Index Terms-Wireless ad hoc network, Gabriel graph, asymptotic probability distribution, the longest edge, poisson point process, topology control.

\section{INTRODUCTION}

A wireless ad hoc network is a collection of wireless devices (transceivers) distributed over a geographic region. Each node is equipped with an omnidirectional antenna and has limited transmission power. A communication session is established either through a single-hop radio transmission if the communication parties are close enough, or through relaying by intermediate devices otherwise. Since they have no need for a fixed infrastructure, wireless ad hoc networks can be flexibly deployed at low cost for varying missions such as decision making in the battlefield, emergency disaster relief, and environmental monitoring.

In wireless ad hoc networks, each node is associated with a maximal transmission radius. The network topology of a wireless ad hoc network is a graph in which two nodes have an edge between them if they are within each other's transmission range. A spanner is a subset of the network topology in which the total cost, e.g., distance or energy consumption, between any pair of nodes is only a constant fact larger than in the original network topology. Hence, spanners are good candidates of virtual backbones. The topics about how to construct and maintain spanners are called topology control. Geometric structures, including Euclidean minimal spanning trees (EMST), relative neighbor

- P.-J. Wan is with the Department of Computer Science, City University of Hong Kong, 83 Tat Chee Avenue, Kowloon, Hong Kong. E-mail:pwan@cs.cityu.edu.hk

- C.-W. Yi is with the Department of Computer Science, National Chiao Tung University, 1001 Ta Hsueh Road, Hsinchu City, Hsinchu 300, Taiwan. E-mail: yi@cs.nctu.edu.tw.

Manuscript received 15 Feb. 2005; revised 1 Sept. 2005; accepted 2 Sept. 2005; published online 28 Nov. 2006.

Recommended for acceptance by I. Stojmenovic.

For information on obtaining reprints of this article, please send e-mail to: tpds@computer.org, and reference IEEECS Log Number TPDS-0100-0205. graphs (RNG), Gabriel graphs (GG), Delauney triangulations (DT), and Yao's graphs (YG), are widely used ingredients for constructing spanners [1], [2], [3]. A topology control algorithm is localized if each node only needs to collect information from few hops neighbors.

In this paper, we study the critical transmission radius for Gabriel graphs. In the GG, two nodes have an edge between them if and only if there is no other node on the disk using the segment of these two nodes as its diameter. Assume all nodes have the same maximal transmission radius $r$. Then, the induced network topology is exactly the $r$-disk graph over the set of nodes $V$, denoted by $G_{r}(V)$. To construct the GG only by 1-hop neighbor information, the transmission radius $r$ should be large enough such that the GG is a subgraph of the $r$-disk graph. Thus, the transmission radius should be not less than the length of the longest edge of the GG. On the other hand, for each node, if it can gather the information of nodes that are not farther than its farthest neighbor in the GG, it can decide all GG edges incident to it. Therefore, the length of the longest edge of the GG is called the critical transmission radius for GGs.

For modeling radio networks, Gilbert [4] proposed a random geometric graph model in which devices are represented by an infinite random point process over the entire plane and two devices are joined by an edge if and only if their distance is at most $r$. For modeling wireless ad hoc networks which consist of finite radio nodes in a bounded geographic region, a bounded (or finite) variant of the Gilbert's model has been used by Gupta and Kumar [5] and others. In this variant, instead of an infinite random point process, the ad hoc device is typically presented by a uniform point process or Poisson point process over a disk or a square by proper scaling. The largest nearest-neighbor link problem 
has been studied by Dette and Henze [6], and the longest edge of the EMST that is related to the connectivity problem has been studied by Penrose [7]. Based on their results, the probability of the event that the length of the longest edge of the EMST is less than $\sqrt{\frac{\ln n+\xi}{\pi n}}$ for some constant $\xi$ is equal to $\exp \left(-e^{-\xi}\right)$ asymptotically. Recently, Kozma et al. [8] proved that the maximal length of an edge in the DT of a uniform $n$-point process in a unit disk is $O\left(\sqrt[3]{\frac{\ln n}{n}}\right)$.

In what follows, $\|x\|$ is the Euclidean norm of a point $x \in \mathbb{R}^{2} .|A|$ is shorthand for 2-dimensional Lebesgue measure (or area) of a measurable set $A \subset \mathbb{R}^{2}$ or the cardinality of a countable set $A$. All integrals considered will be Lebesgue integrals. The topological boundary of a set $A \subset \mathbb{R}^{2}$ is denoted by $\partial A$. The disk of radius $r$ centered at $x$ is denoted by $B(x, r)$. The special unit-area disk centered at the origin is denoted by D. For any set $S$ and positive integer $k$, the $k$-fold Cartesian product of $S$ is denoted by $S^{k}$. An event is said to be asymptotically almost sure (abbreviated by a.a.s.) if it occurs with a probability that converges to one as $n \rightarrow \infty$. An event is said to be asymptotically almost rare (abbreviated by a.a.r.) if it occurs with a probability that converges to zero as $n \rightarrow \infty$. The symbols $O, o, \sim$ always refer to the limit $n \rightarrow \infty$. To avoid trivialities, we tacitly assume $n$ to be sufficiently large if necessary. For simplicity of notation, the dependence of sets and random variables on $n$ will be frequently suppressed.

The remainder of this paper is organized as follows: In Section 2, we give a brief review of our main results. In Section 3, we present several useful geometric results and integrals. In Section 4, we derive the asymptotic length of the longest edge. In Section 5, we drive the asymptotic expected number of long edges. In Section 6, we drive the asymptotic distribution of the length of the longest edge. We summarize this paper in Section 7.

\section{Main Results}

In this paper, we assume a wireless ad hoc network is represented by a Poisson point process over a unit-area disk with mean $n$, denoted by $\mathcal{P}_{n}$, and all nodes have the same maximal transmission radius $r_{n}$ which is a function of $n$. We use $G\left(\mathcal{P}_{n}\right)$ to denote the Gabriel graph over $\mathcal{P}_{n}$. For simplicity, the edges of GGs are called Gabriel edges. If $G$ is a geometric graph, we use $\lambda(G)$ to denote the maximal length of an edge of $G$ and $N(G, l)$ to denote the number of edges of $G$ whose length is at least $l$. Our first main result is the next theorem.

Theorem 1. For any constant $\varepsilon>0$, we have

$$
\lim _{n \rightarrow \infty} \operatorname{Pr}\left[(1-\varepsilon) 2 \sqrt{\frac{\ln n}{\pi n}} \leq \lambda\left(G\left(\mathcal{P}_{n}\right)\right) \leq(1+\varepsilon) 2 \sqrt{\frac{\ln n}{\pi n}}\right]=1
$$

According to Theorem 1, if each node sets its maximal transmission radius to $r_{n}=\beta \sqrt{\frac{\ln n}{\pi n}}$ for some constant $\beta$, then the $r_{n}$-disk graph over $\mathcal{P}_{n}$ a.a.s. contains the GG if $\beta>2$, and on the contrary, the $r_{n}$-disk graph a.a.r. contains the GG if $\beta<2$. Therefore, $\beta=2$ is the threshold for constructing the GG by 1-hop information. For reference, we remark that $\beta=1$ is the threshold for the $r_{n}$-disk graph being connected [5], [9].
Now, we assume $r_{n}=2 \sqrt{\frac{\ln n+\xi}{\pi n}}$ for some constant $\xi$. For a given $\xi$, we call the edge whose length is not less than $r_{n}$ a long edge. The next theorem gives us the asymptotic expectation of the number of long Gabriel edges.

Theorem 2. For the expectation of the number of long Gabriel edges, we have

$$
\lim _{n \rightarrow \infty} \mathbf{E}\left[N\left(G\left(\mathcal{P}_{n}\right), 2 \sqrt{\frac{\ln n+\xi}{n \pi}}\right)\right]=2 e^{-\xi} .
$$

Since $\operatorname{Pr}[X=0]=1-\operatorname{Pr}[X \geq 1] \geq 1-\mathbf{E}[X]$ for any nonnegative integer value $\mathrm{RV} X$,

$$
\begin{aligned}
& \operatorname{Pr}\left[\lambda\left(G\left(\mathcal{P}_{n}\right)\right)<2 \sqrt{\frac{\ln n+\xi}{\pi n}}\right] \\
& =\operatorname{Pr}\left[N\left(G\left(\mathcal{P}_{n}\right), 2 \sqrt{\frac{\ln n+\xi}{n \pi}}\right)=0\right] \\
& \geq 1-\mathbf{E}\left[N\left(G\left(\mathcal{P}_{n}\right), 2 \sqrt{\frac{\ln n+\xi}{n \pi}}\right)\right] \\
& \sim 1-2 e^{-\xi} .
\end{aligned}
$$

Therefore,

$$
\lim _{\xi \rightarrow \infty} \lim _{n \rightarrow \infty} \operatorname{Pr}\left[\lambda\left(G\left(\mathcal{P}_{n}\right)\right)<2 \sqrt{\frac{\ln n+\xi}{\pi n}}\right]=1,
$$

and $\xi \rightarrow \infty$ is an a.a.s. sufficient condition for $\lambda\left(G\left(\mathcal{P}_{n}\right)\right)<$ $2 \sqrt{\frac{\ln n+\xi}{\pi n}}$. In the next theorem, we give the asymptotic probability distribution of the number of long Gabriel edges, and that implies the asymptotic probability distribution of the length of the longest edge.

Theorem 3. For any constant $\xi$, the total number of Gabriel edges whose lengths are at least $2 \sqrt{\frac{\ln n+\xi}{\pi n}}$ is asymptotically Poisson with mean $2 e^{-\xi}$.

Since

$$
\begin{aligned}
& \operatorname{Pr}\left[\lambda\left(G\left(\mathcal{P}_{n}\right)\right)<2 \sqrt{\frac{\ln n+\xi}{\pi n}}\right] \\
& =\operatorname{Pr}\left[N\left(G\left(\mathcal{P}_{n}\right), 2 \sqrt{\frac{\ln n+\xi}{n \pi}}\right)=0\right],
\end{aligned}
$$

according to Theorem 3, we have

$$
\lim _{n \rightarrow \infty} \operatorname{Pr}\left[\lambda\left(G\left(\mathcal{P}_{n}\right)\right)<2 \sqrt{\frac{\ln n+\xi}{\pi n}}\right]=\exp \left(-2 e^{-\xi}\right) .
$$

\section{Preliminaries}

In this section, we shall give some definitions and lemmas that will be used to prove our main results.

\subsection{Geometry Preliminaries}

The results in this section are purely geometric, with no probabilistic content. Let ID denote a unit-area disk, $R_{0}=\frac{1}{\sqrt{\pi}}$ 


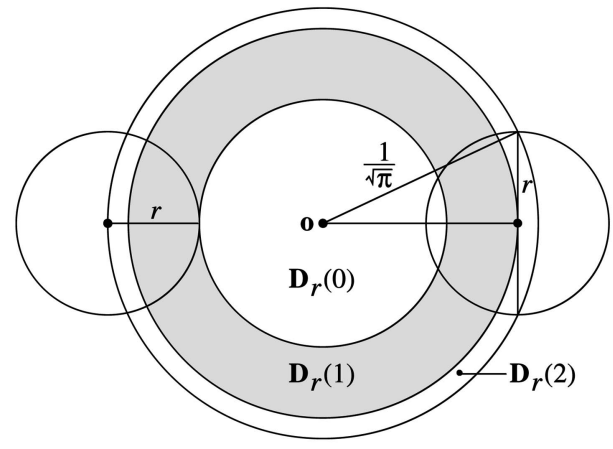

Fig. 1. The partition of the unit-area disk $\mathbb{D}$.

denote the radius of $\mathbb{D}$, and $\mathbf{o}$ denote the origin. Without loss of generality, we assume $\mathbb{D}$ is centered at the origin. For a given transmission radius $r$, the unit-area disk $\mathbb{D}$ is partitioned into $\mathbb{D}_{r}(0), \mathbb{D}_{r}(1)$, and $\mathbb{D}_{r}(2)$ as shown in Fig. 1 : $\mathbb{D}_{r}(0)$ is the disk of radius $\frac{1}{\sqrt{\pi}}-r$ centered at the origin, $\mathbb{D}_{r}(1)$ is the annulus of radii $\frac{1}{\sqrt{\pi}}-r$ and $\sqrt{\frac{1}{\pi}-r^{2}}$ centered at the origin, and $\mathbb{D}_{r}(2)$ is the annulus of radii $\sqrt{\frac{1}{\pi}-r^{2}}$ and $\frac{1}{\sqrt{\pi}}$ centered at the origin. Then,

$$
\left|\mathbb{D}_{r}(0)\right|=(1-\sqrt{\pi} r)^{2},\left|\mathbb{D}_{r}(1)\right|=2 \pi r\left(\frac{1}{\sqrt{\pi}}-r\right),
$$$$
\text { and }\left|\mathbb{D}_{r}(2)\right|=\pi r^{2} \text {. }
$$

If $R$ is a positive number, for any finite set of nodes $V=\left\{x_{1}, \cdots, x_{k}\right\}$, we use $G_{R}\left(x_{1}, \cdots, x_{k}\right)$ or $G_{R}(V)$ to denote the $R$-disk graph over $\left\{x_{1}, \cdots, x_{k}\right\}$ in which there is an edge between two nodes if and only if their Euclidean distance is at most $R$. For any positive integers $k$ and $m$ with $1 \leq m \leq k$ and any positive number $R$, let $C_{k m}(R)$ denote the set of $\left(x_{1}, \cdots, x_{k}\right) \in \mathbb{D}^{k}$ satisfying that $G_{2 R}\left(x_{1}, \cdots, x_{k}\right)$ has exactly $m$ connected components. For any two points $u$ and $v$, let $D_{u v}$ denote the disk with the segment $u v$ as a diameter, i.e., $D_{u v}=B\left(\frac{u+v}{2}, \frac{\|u-v\|}{2}\right)$. We have

$$
\left|D_{u v}\right|=\frac{1}{4} \pi\|u-v\|^{2} .
$$

For any $r=\left(r_{1}, \cdots, r_{k}\right) \in\left(0, R_{0}\right)^{k}$ and $x=\left(x_{1}, \cdots, x_{k}\right) \in$ $\mathrm{D}^{k}, B\left(x_{1}, r_{1}\right), \cdots, B\left(x_{k}, r_{k}\right)$ are called feasible if

$$
\left\|x_{i}-x_{j}\right\| \geq \sqrt{\left|r_{i}^{2}-r_{j}^{2}\right|}
$$

for any $i \neq j$ and $\left\|x_{i}-\mathbf{o}\right\| \leq \sqrt{R_{0}^{2}-r_{i}^{2}}$ for any $i$. We remark that $\left\|x_{i}-\mathbf{o}\right\| \leq \sqrt{R_{0}^{2}-r_{i}^{2}}$ if and only if $x_{i} \in \mathbb{D}_{r_{i}}(0) \cup \mathbb{D}_{r_{i}}(1)$, and $\left\|x_{i}-x_{j}\right\| \geq \sqrt{\left|r_{i}^{2}-r_{j}^{2}\right|}$ if and only if both disks $B\left(x_{i}, r_{i}\right)$ and $B\left(x_{j}, r_{j}\right)$ do not contain each other's diameter. Let $\mathbf{1}_{x}^{r}$ be an indicator such that $\mathbf{1}_{x}^{r}=1$ if $B\left(x_{1}, r_{1}\right), \cdots, B\left(x_{k}, r_{k}\right)$ are feasible, and $\mathbf{1}_{x}^{r}=0$ otherwise. In what follows, we only consider feasible disk sets. We use $\nu_{r}(x)$ to denote the area of $\bigcup_{i=1}^{k} B\left(x_{i}, r_{i}\right) \cap \mathbb{D}$, and sometimes by slightly abusing the notation, to denote the union region itself. If $k=1$, for $x \in \mathbb{D}_{r}(0), \nu_{r}(x)=\pi r^{2}$ and, for $x \in \mathbb{D}_{r}(1), \nu_{r}(x) \geq \frac{1}{2} \pi r^{2}$.

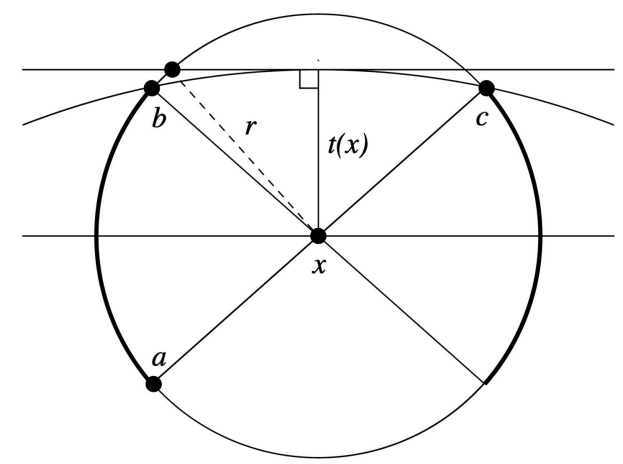

Fig. 2. If $x \in \mathbb{D}_{r}(1)$, then $\theta(x, r)=2\llcorner a x b$.

Furthermore, the following lemma proved in [10] gives a tighter lower bound for $\nu_{r}(x)$.

Lemma 4. For any $x \in \mathbb{D}_{r}(1)$,

$$
\nu_{r}(x) \geq \frac{1}{2} \pi r^{2}+\left(\frac{1}{\sqrt{\pi}}-\|x\|\right) r .
$$

The next lemma gives a lower bound for the area of the $r$-neighborhood of more than one nodes.

Lemma 5. Let $R \leq \frac{1}{100} R_{0}, c=0.03, x=\left(x_{1}, \cdots, x_{k}\right) \in \mathbb{D}^{k}$, and $r=\left(r_{1}, \cdots, r_{k}\right) \in\left[\frac{1}{2} R, R\right]^{k}$. Assume $x_{1}$ has the largest norm among $x_{1}, \cdots, x_{k}$, and $\left\|x_{i}-x_{j}\right\| \leq 2 R$ if and only if $|i-j| \leq 1$. If $\mathbf{1}_{x}^{r}=1$, then

$$
\nu_{r}(x) \geq \nu_{r_{1}}\left(x_{1}\right)+c R \sum_{I=1}^{k-1}\left\|x_{I+1}-x_{i}\right\| .
$$

The proof of Lemma 5 is given in the Appendix.

Corollary 6. Assume $R \leq \frac{1}{100} R_{0}$ and $c=0.03$. If $x=\left(x_{1}, \cdots\right.$, $\left.x_{k}\right) \in C_{k 1}(R), r=\left(r_{1}, \cdots, r_{k}\right) \in\left[\frac{1}{2} R, R\right]^{k}, \mathbf{1}_{x}^{r}=1$, and $x_{1}$ has the largest norm among $x_{1}, \cdots, x_{k}$, then

$$
\nu_{r}(x) \geq \nu_{r_{1}}\left(x_{1}\right)+c R \max _{2 \leq i \leq k}\left\|x_{i}-x_{1}\right\| .
$$

Proof. Without loss of generality, we assume that $\left\|x_{k}-x_{1}\right\|$ achieves $\max _{2 \leq i \leq k}\left\|x_{i}-x_{1}\right\|$. Let $P$ be a min-hop path between $x_{1}$ and $x_{k}$ in $G_{2 R}\left(x_{1}, x_{2}, \cdots, x_{k}\right)$ and $t$ be the total length of $P$. Then, every pair of nodes in $P$ that are not adjacent nodes in $P$ are separated by a distance of more than $R$. Thus, by applying Lemma 5 to the nodes in $P$, we obtain

$$
\nu_{\left(\left\{r_{i} \mid x_{i} \in P\right\}\right)}\left(\left\{x_{i} \mid x_{i} \in P\right\}\right) \geq \nu_{r_{1}}\left(x_{1}\right)+c R t .
$$

Since $\nu_{r}(x) \geq \nu_{\left(\left\{r_{i} \mid x_{i} \in P\right\}\right)}\left(\left\{x_{i} \mid x_{i} \in P\right\}\right)$ and $t \geq\left\|x_{k}-x_{1}\right\|$, the corollary follows.

For $x \in \mathbb{D}$ and $r \in \mathbb{R}$, let $\theta(x, r)$ denote the (total) central angle corresponding to the portion of $\partial B(x, r)$ in which, if a diameter of $B(x, r)$ has endpoints, it is fully contained in $\mathbb{D}$. For example, in Fig. 2, $x \in \mathbb{D}_{r}(1), b$ and $c$ are intersection points of $\partial B(x, r)$ and $\partial \mathbb{D}$, and segment $a c$ is a diameter of $B(x, r)$. Then, $\theta(x, r)=2 \measuredangle a x b$. If $x \in \mathbb{D}_{r}(1)$, we use $t(x)$ to denote the distance between $x$ and $\partial \mathbb{D}$. We have 


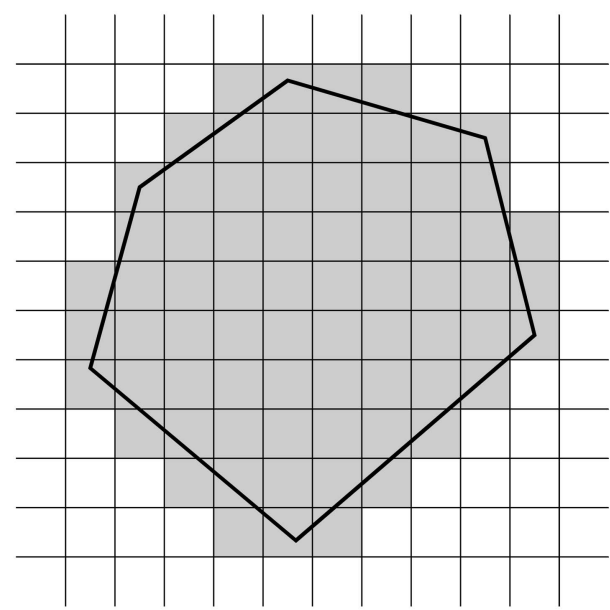

Fig. 3. A polyquadrate is a collection of grids that intersect with a polygon.

$$
\begin{aligned}
& \theta(x, r)=2 \pi, \text { if } x \in \mathbb{D}_{r}(0) \\
& \theta(x, r) \leq 4 \arcsin \frac{t(x)}{r} \leq 4 \frac{\pi}{2} \frac{t(x)}{r}, \text { if } x \in \mathbb{D}_{r}(1) \\
& \theta(x, r)=0, \text { if } x \in \mathbb{D}_{r}(2) .
\end{aligned}
$$

An $\varepsilon$-tessellation is to divide the plane by vertical and horizontal lines into a grid in which each cell is with width $\varepsilon$. Without loss of generality, we assume the origin is a corner in the grid. A collection of grid cells intersecting with a polygon or a convex compact set is called a polyquadrate. For example, in Fig. 3, the shaded grid cells form a polyquadrate. The horizontal span of a polyquadrate is the horizontal distance measured by the number of grid cells from the left to the right. The vertical span of a polyquadrate is with similar definite, but for the vertical distance.

Lemma 7. If $S$ consists of $m$ grid cells and $n$ is a constant, the number of polyquadrates with span less than $n$ and intersecting with $S$ is $\Theta(m)$.

Proof. Since $n$ is a constant, the number of polyquadrates that have spans less than $n$ and contain a specified grid cell is also constant. Since $S$ consists of $m$ grid cells, the lemma follows.

\subsection{Extremes of a Collection of Poisson RVs}

The next lemma gives an a.a.s. upper bound and lower bound of a collection of Poisson RVs.

Lemma 8. Let $Y_{i}$ be a Poisson $R V$ with rate $\lambda_{i}$ for $i=1, \cdots, I_{n}$ and let $c>0$ and $\beta>0$ be constants. Suppose $I_{n}=\Theta\left(\left(\frac{n}{\ln n}\right)^{c}\right)$. If $\beta \geq c$ and $\lambda_{i} \geq \beta \ln n$, we have

$$
\lim _{n \rightarrow \infty} \operatorname{Pr}\left[\min _{i=1}^{I_{n}} Y_{i}>0\right]=1
$$

If $Y_{1}, Y_{2}, \cdots, Y_{I_{n}}$ are independent, $\beta \in(0, c)$, and $\lambda_{i} \leq \beta \ln n$, we have

$$
\lim _{n \rightarrow \infty} \operatorname{Pr}\left[\min _{i=1}^{I_{n}} Y_{i}=0\right]=1
$$

Proof. Let $X_{i}=1_{\left\{Y_{i}=0\right\}}$ and $X=\sum_{i=1}^{I_{n}} X_{i}$. We have

$$
\begin{aligned}
& \operatorname{Pr}\left[\min _{i=1}^{I_{n}} Y_{i}>0\right]=\operatorname{Pr}[X=0]=1-\operatorname{Pr}[X>0] \\
& \geq 1-\mathbf{E}[X]=1-\sum_{i=1}^{I_{n}} \mathbf{E}\left[X_{i}\right]=1-\sum_{i=1}^{I_{n}} \operatorname{Pr}\left[Y_{i}=0\right] .
\end{aligned}
$$

Therefore, if $\beta \geq c$ and $\lambda_{i} \geq \beta \ln n$,

$$
\begin{aligned}
& \operatorname{Pr}\left[\min _{i=1}^{I_{n}} Y_{i}>0\right] \geq 1-\sum_{i=1}^{I_{n}} \operatorname{Pr}\left[Y_{i}=0\right]=1-\sum_{i=1}^{I_{n}} e^{-\lambda_{i}} \\
& \geq 1-\sum_{i=1}^{I_{n}} e^{-\beta \ln n}=1-\Theta\left(\left(\frac{n}{\ln n}\right)^{c}\right) e^{-\beta \ln n} \\
& =1-\Theta\left(\frac{n^{c-\beta}}{\ln ^{c} n}\right) \sim 1 .
\end{aligned}
$$

If $Y_{1}, Y_{2}, \cdots, Y_{I_{n}}$ are independent, we have

$$
\begin{aligned}
& \operatorname{Pr}\left[\min _{i=1}^{I_{n}} Y_{i}=0\right]=1-\operatorname{Pr}\left[\min _{i=1}^{I_{n}} Y_{i}>0\right]=1-\prod_{1=i}^{I_{n}} \operatorname{Pr}\left[Y_{i}>0\right] \\
& =1-\prod_{1=i}^{I_{n}}\left(1-\operatorname{Pr}\left[Y_{i}=0\right]\right) \geq 1-\prod_{1=i}^{I_{n}} e^{-\operatorname{Pr}\left[Y_{i}=0\right]}=1-e^{-\sum_{i=1}^{I_{n}} \operatorname{Pr}\left[Y_{i}=0\right]} .
\end{aligned}
$$

Therefore, if $\beta \in(0, c)$ and $\lambda_{i} \leq \beta \ln n$,

$$
\begin{aligned}
& \operatorname{Pr}\left(\min _{1 \leq i \leq I_{n}} Y_{i}=0\right) \geq 1-e^{-\sum_{i=1}^{I_{n}} \operatorname{Pr}\left(Y_{i}=0\right)}=1-e^{-\sum_{i=1}^{I_{n}} e^{-\lambda_{i}}} \\
& \geq 1-e^{-\sum_{i=1}^{I_{n}} e^{-\beta \ln n}}=1-e^{-\Theta\left(\left(\frac{n}{\ln n}\right)^{c}\right) e^{-\beta \ln n}}=1-e^{-\Theta\left(\frac{n^{c-\beta}}{\ln { }^{c} n}\right)} \sim 1 .
\end{aligned}
$$

\subsection{Palm Theory and Brun's Sieve}

Here, we state the Palm theory [11], [12] on the Poisson point process.

Theorem 9. Let $n>0$. Suppose $k$ is a positive integer and $h(\mathcal{Y}, \mathcal{X})$ is a bounded measurable function defined on all pairs of the form $(\mathcal{Y}, \mathcal{X})$ with $\mathcal{X} \subset \mathbb{R}^{2}$ being a finite subset and $\mathcal{Y}$ being a subset of $\mathcal{X}$ satisfying $h(\mathcal{Y}, \mathcal{X})=0$ except when $\mathcal{Y}$ has $k$ elements. Then,

$$
\mathbf{E}\left[\sum_{\mathcal{Y} \subseteq \mathcal{P}_{n}} h\left(\mathcal{Y}, \mathcal{P}_{n}\right)\right]=\frac{n^{k}}{k !} \mathbf{E}\left[h\left(\mathcal{X}_{k}, \mathcal{X}_{k} \cup \mathcal{P}_{n}\right)\right],
$$

where the sum on the left-hand side is over all subsets $\mathcal{Y}$ of the random Poisson point set $\mathcal{P}_{n}$ and, on the right-hand side, the set $\mathcal{X}_{k}$ is a binomial process with $k$ nodes, independent of $\mathcal{P}_{n}$.

The next theorem is called Brun's sieve, which is a traditional approach to the Poisson process and will be used to prove Theorem 3.

Theorem 10. Assume $m(n)$ is a nonnegative integer random variable. Let $B_{1}, \cdots, B_{m(n)}$ be events, $Y$ be the number of $B_{i}$ that holds, and

$$
S^{(j)}=\sum_{\left\{i_{1}, \cdots, i_{j}\right\} \subseteq\{1, \cdots, q m(n)\}} \operatorname{Pr}\left[B_{i_{1}} \wedge \cdots \wedge B_{i_{j}}\right] .
$$

Suppose there is a constant $\mu$ such that, for every fixed $k$,

$$
\mathbf{E}\left[S^{(j)}\right] \sim \frac{1}{j !} \mu^{j}
$$

Then, $Y$ is also asymptotically Poisson with mean $\mu$. 
Proof. To prove this, we need to show that, for any $k$,

$$
\operatorname{Pr}[Y=k] \rightarrow \frac{\mu^{k}}{k !} e^{-\mu} .
$$

For any $i \geq j \geq 1$, let

$$
S_{i}^{(j)}=\sum_{\left\{i_{1}, \cdots, i_{j}\right\} \subseteq\{1, \cdots, m(n)\}} \operatorname{Pr}\left[B_{i_{1}} \wedge \cdots \wedge B_{i_{j}} \mid m(n)=i\right] .
$$

For convenience, let $S^{(0)}=1$ and $S_{i}^{(0)}=1$. In addition, note that, for any $j \geq 1$,

$$
\mathbf{E}\left[S^{(j)}\right]=\sum_{i=j}^{\infty} S_{i}^{(j)} \operatorname{Pr}[m(n)=i] .
$$

According to the inclusion-exclusion principle, we have

$$
\begin{aligned}
\operatorname{Pr}[Y=0] & =1-S^{(1)}+S^{(2)}-\cdots \\
& =\sum_{i=0}^{\infty}\left(\sum_{j=0}^{i}(-1)^{j} S_{i}^{(j)}\right) \operatorname{Pr}[m(n)=i] \\
& =\left(\sum_{j=0}^{\infty}(-1)^{j}\left(\sum_{i=j}^{\infty} S_{i}^{(j)} \operatorname{Pr}[m(n)=i]\right)\right) \\
& =\sum_{j=0}^{\infty}(-1)^{j} \mathbf{E}\left[S^{(j)}\right] .
\end{aligned}
$$

In the general form, we have

$$
\begin{aligned}
\operatorname{Pr}[Y=k] & =\left(\begin{array}{l}
k \\
k
\end{array}\right) S^{(k)}-\left(\begin{array}{c}
k+1 \\
k
\end{array}\right) S^{(k+1)}+\cdots \\
& =\sum_{i=k}^{\infty}\left(\sum_{j=k}^{i}(-1)^{j-k}\left(\begin{array}{c}
j \\
j-k
\end{array}\right) S_{i}^{(j)}\right) \operatorname{Pr}[m(n)=i] \\
& =\left(\sum_{j=k}^{\infty}(-1)^{j-k}\left(\begin{array}{c}
j \\
j-k
\end{array}\right)\left(\sum_{i=j}^{\infty} S_{i}^{(j)} \operatorname{Pr}[m(n)=i]\right)\right) \\
& =\sum_{j=k}^{\infty}(-1)^{j-k}\left(\begin{array}{c}
j \\
j-k
\end{array}\right) \mathbf{E}\left[S^{(j)}\right] .
\end{aligned}
$$

Then, following the proof outline given in [13], Chapter 8, the theorem can be proved.

Here, we remark that Theorem 10, in which the number of events is a random variables is an extension to the traditional Brun's sieve described in [13] which is an implication of the Bonferroni inequalities.

\subsection{Integral Preliminaries}

First, we introduce a technique to obtain the Jacobian determinant in the change of variables. Assume a tree topology is fixed over $x_{1}, x_{2}, \cdots, x_{k} \in \mathbb{R}^{2}$. Without loss of generality, we may assume $\left(x_{k-1}, x_{k}\right)$ is one of edges. Let $z_{k-1}=\frac{1}{2}\left(x_{k-1}+x_{k}\right), r=\frac{1}{2}\left\|x_{k}-x_{k-1}\right\|$, and $\theta$ be the slope of $x_{k-1} x_{k}$. For $1 \leq i \leq k-2$, we use $p\left(x_{i}\right)$ to denote $x_{i}$ 's parent in the tree rooted at $x_{k}$, and let $z_{i}=\frac{1}{2}\left(x_{i}+p\left(x_{i}\right)\right)$. Then,

$$
\begin{aligned}
& \left|\frac{\partial\left(x_{1}, \cdots, x_{k-1}, x_{k}\right)}{\partial\left(z_{1}, \cdots, z_{k-1}, r, \theta\right)}\right|=\left|\frac{\partial\left(x_{1}+p\left(x_{1}\right), \cdots, x_{k-1}+p\left(x_{k-1}\right), x_{k}\right)}{\partial\left(z_{1}, \cdots, z_{k-1}, r, \theta\right)}\right| \\
& =4^{k-1}\left|\frac{\partial\left(\frac{x_{1}+p\left(x_{1}\right)}{2}, \cdots, \frac{x_{k-1}+p\left(x_{k-1}\right)}{2}, x_{k}\right)}{\partial\left(z_{1}, \cdots, z_{k-1}, r, \theta\right)}\right| \\
& =4^{k-1}\left|\frac{\partial\left(z_{1}, \cdots, z_{k-1}, x_{k}-z_{k-1}\right)}{\partial\left(z_{1}, \cdots, z_{k-1}, r, \theta\right)}\right| \\
& =4^{k-1}\left|\begin{array}{ccccc}
I_{2} & \cdots & 0 & 0 \\
\vdots & \ddots & \vdots & \vdots \\
0 & \cdots & I_{2} & & 0 \\
0 & \cdots & 0 & \cos \theta & -r \sin \theta \\
\sin \theta & r \cos \theta
\end{array}\right| \\
& =4^{k-1} r \text {. }
\end{aligned}
$$

Now, we give several lemmas about the limits of integrals, but leave their proofs in the Appendix.

Lemma 11. Let $r_{\xi}=2 \sqrt{\frac{\ln n+\xi}{\pi n}}$ for some constant $\xi$, and either $R_{n}=3 \sqrt{\frac{\ln n}{\pi n}}$ or $R_{n}=2 \sqrt{\frac{\ln n+\xi_{n}}{\pi n}}$ with $\xi_{n}=o(\ln n)$ and $\xi_{n} \rightarrow \infty$.

Then,

$$
\frac{n^{2}}{2} \int_{r=\frac{r_{\xi}}{2}}^{\frac{R_{n}}{2}} \int_{z \in \mathbb{D}} e^{-n \nu_{r}(z)} 4 r \theta(z, r) d z d r \sim 2 e^{-\xi} .
$$

In the remainder of this section, we always assume $r_{\xi}=$ $2 \sqrt{\frac{\ln n+\xi}{\pi n}}$ for some constant $\xi$, and $R_{n}=2 \sqrt{\frac{\ln n+\xi_{n}}{\pi n}}$ with $\xi_{n}=$ $o(\ln n)$ and $\xi_{n} \rightarrow \infty$.

Lemma 12. For any fixed integer $k \geq 2$,

$\left(\frac{n^{2}}{2}\right)^{k} \int_{r \in\left[\frac{r_{\xi}}{2}, \frac{\left.R_{n}\right]^{k}}{2}\right]^{r^{2}}} \int_{z \in C_{k 1}\left(\frac{R_{n}}{2}\right)} \mathbf{1}_{z}^{r} e^{-n \nu_{r}(z)} \prod_{i=1}^{k} 4 r_{i} \theta\left(z_{i}, r_{i}\right) d z_{i} d r_{i}=o(1)$

Lemma 13. For any fixed integers $2 \leq m<k$,

$$
\begin{aligned}
& \left(\frac{n^{2}}{2}\right)^{k} \int_{r \in\left[\frac{\xi_{2}}{2}, \frac{R_{n}}{2}\right]^{k}} \int_{z \in C_{k m}\left(\frac{R_{n}}{2}\right)} \mathbf{1}_{z}^{r} e^{-n \nu_{r}(z)} \prod_{i=1}^{k} 4 r_{i} \theta\left(z_{i}, r_{i}\right) d z_{i} d r_{i} \\
& =o(1) .
\end{aligned}
$$

Lemma 14. For any fixed integer $k \geq 2$,

$$
\begin{aligned}
& \left(\frac{n^{2}}{2}\right)^{k} \int_{r \in\left[\frac{r_{2}}{2}, \frac{R_{n}}{2}\right]^{k}} \int_{z \in C_{k k}\left(\frac{R n}{2}\right)} e^{-n \nu_{r}(z)} \prod_{i=1}^{k} 4 r_{i} \theta\left(z_{i}, r_{i}\right) d z_{i} d r_{i} \\
& \quad \sim\left(2 e^{-\xi}\right)^{k} .
\end{aligned}
$$

Lemma 15. For any constant $m \geq 3$ and $\frac{m}{2} \geq t \geq i \geq 1$, let $S^{i j}$ denote the set of $\left(z_{1}, z_{2}, \cdots, z_{m-t}\right) \in C_{(m-t) 1}\left(\frac{R_{n}}{2}\right)$ satisfying that $z_{i}$ is the one with largest norm and $z_{j}$ is the one with smallest norm among $z_{1}, \cdots, z_{k}$. Then, 


$$
\begin{aligned}
& n^{m} \int_{r \in\left[\frac{r_{\xi}}{2}, \frac{R_{n}}{2}\right]^{t}} \int_{z \in S^{i j}} e^{-n\left(\nu_{r_{i}}\left(z_{i}\right)+c R_{n}\left\|z_{i}-z_{j}\right\|\right)}\left(\prod_{k=1}^{t} r_{k} \theta\left(z_{k}, r_{k}\right) d r_{k}\right) \\
& \left(\prod_{k=1}^{m-t} d z_{k}\right) \sim o(1) .
\end{aligned}
$$

\section{Asymptotic Length OF the Longest Edge}

This section is dedicated to the proof of Theorem 1.

\subsection{Lower Bounds for the Longest Edge Length}

In this section, we are going to prove the following lemma that gives lower bounds for the length of the longest Gabriel edge.

Lemma 16. For any constant $\beta \in(0,2)$,

$$
\lim _{n \rightarrow \infty} \operatorname{Pr}\left[\lambda\left(G\left(\mathcal{P}_{n}\right)\right) \geq \beta \sqrt{\frac{\ln n}{\pi n}}\right]=1 .
$$

Assume $\beta_{1}$ and $\beta_{2}$ are positive constants, and $R_{1}$ and $R_{2}$ are given by $n \pi R_{1}^{2}=\beta_{1} \ln n$ and $n \pi R_{2}^{2}=\beta_{2} \ln n$, respectively. Choose $\beta_{1}, \beta_{2}$ such that $\max \left(1, \beta^{2}\right)<\beta_{1}<\beta_{2}<4$ and $\frac{\pi^{2}}{c^{2}}\left(1-\frac{R_{1}}{R_{2}}\right)<1$. Here, $c$ is given by Lemma 5 . It follows that $\frac{1}{2} R_{2} \leq R_{1} \leq R_{2}$. Consider a $\left(3 \sqrt{\frac{\ln n}{n \pi}}\right)$-tessellation and the grid cells within $\mathrm{DD}$. Let $I_{n}$ denote the number of cells fully contained in $\mathrm{D}$. Here, $I_{n}=O\left(\frac{n}{\ln n}\right)$. For each cell fully contained in $\mathbb{D}$, we draw a disk with radius $\frac{1}{2} \sqrt{\frac{\ln n}{n \pi}}$ at the center of the cell. For $1 \leq i \leq I_{n}$, let $E_{i}$ be the event that there exist Gabriel edges whose midpoints are on the $i$ th disk and whose lengths are between $R_{1}$ and $R_{2}$. Hence,

$$
\operatorname{Pr}\left[\lambda\left(G\left(\mathcal{P}_{n}\right)\right) \geq \beta \sqrt{\frac{\ln n}{\pi n}}\right] \geq \operatorname{Pr}\left[\text { at least one } E_{i} \text { occurs }\right] .
$$

We have that $E_{1}, \cdots, E_{I_{i}}$ are identical. If $o_{i}$ denotes the center of the $i$ th disk, $u$ and $v$ are two points such that their midpoint is on the $i$ th disk, and the distance between them is between $R_{1}$ and $R_{2}$, then, for any point $w \in D_{u v}$, we have

$$
\begin{aligned}
\left\|w-o_{i}\right\| & \leq\left\|w-\frac{1}{2}(u+v)\right\|+\left\|o_{i}-\frac{1}{2}(u+v)\right\| \\
& \leq \frac{1}{2} R+\frac{1}{2} \sqrt{\frac{\ln n}{n \pi}}<\frac{3}{2} \sqrt{\frac{\ln n}{n \pi}} .
\end{aligned}
$$

Therefore, $u, v$, and $D_{u v}$ are contained in the $i$ th cell. (See Fig. 4.) Hence, $E_{1}, \cdots, E_{I_{i}}$ are independent. Then,

$$
\begin{aligned}
\operatorname{Pr}\left[\text { none of } E_{i} \text { occurs }\right] & =\left(1-\operatorname{Pr}\left[E_{1}\right]\right)^{I_{n}}=e^{I_{n} \ln \left(1-\operatorname{Pr}\left[E_{1}\right]\right)} \\
& \leq e^{-I_{n} \operatorname{Pr}\left(E_{1}\right)}
\end{aligned}
$$

If $I_{n} \operatorname{Pr}\left(E_{1}\right) \rightarrow \infty$, we may have

$$
\operatorname{Pr}\left[\lambda\left(G\left(\mathcal{P}_{n}\right)\right) \geq \beta \sqrt{\frac{\ln n}{\pi n}}\right] \rightarrow 1 .
$$

In the following, we shall prove that $I_{n} \operatorname{Pr}\left(E_{1}\right) \rightarrow \infty$.

Let $A$ denote the first disk. Assume $V$ is a point set and $Y \subset V$. Let $h_{1}(Y, V)$ denote a function such that $h_{1}(Y=$ $\left.\left\{x_{1}, x_{2}\right\}, V\right)=1$ only if $\frac{1}{2}\left(x_{1}+x_{2}\right) \in A, R_{1} \leq\left\|x_{1}-x_{2}\right\| \leq R_{2}$,

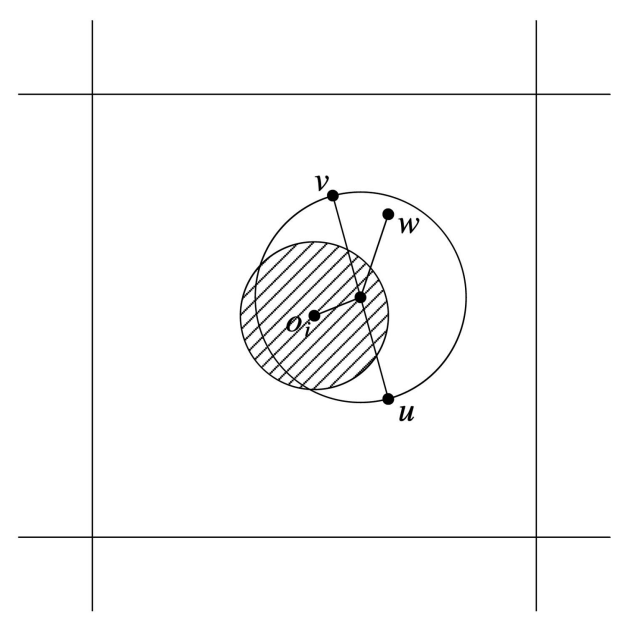

Fig. 4. $D_{u v}$ is fully contained in the cell.

and there is no other node of $V$ in the disk area $D_{x_{1} x_{2}}$; otherwise, $h_{1}(Y, V)=0$. Hence, $E_{1}$ is the event that there exist two nodes $X, Y \in \mathcal{P}_{n}$ such that $h_{1}\left(\{X, Y\}, \mathcal{P}_{n}\right)=1$. In the remainder of this section, we use $X_{1}, X_{2}, X_{3}$, and $X_{4}$ to denote independent random points with uniform distribution over ID and independent of $\mathcal{P}_{n}$. Let $F_{1}$ be the event that

$$
h_{1}\left(\left\{X_{1}, X_{2}\right\},\left\{X_{1}, X_{2}\right\} \cup \mathcal{P}_{n}\right)=1,
$$

$F_{2}$ be the event that

$$
\begin{aligned}
& h_{1}\left(\left\{X_{1}, X_{2}\right\},\left\{X_{1}, X_{2}, X_{3}\right\} \cup \mathcal{P}_{n}\right) \\
& \cdot h_{1}\left(\left\{X_{1}, X_{3}\right\},\left\{X_{1}, X_{2}, X_{3}\right\} \cup \mathcal{P}_{n}\right)=1,
\end{aligned}
$$

and $F_{3}$ be the event that

$$
\begin{aligned}
& h_{1}\left(\left\{X_{1}, X_{2}\right\},\left\{X_{1}, X_{2}, X_{3}, X_{4}\right\} \cup \mathcal{P}_{n}\right) \\
& \cdot h_{1}\left(\left\{X_{3}, X_{4}\right\},\left\{X_{1}, X_{2}, X_{3}, X_{4}\right\} \cup \mathcal{P}_{n}\right)=1 .
\end{aligned}
$$

We claim that

$$
\operatorname{Pr}\left[E_{1}\right] \geq \frac{n^{2}}{2 !} \operatorname{Pr}\left[F_{1}\right]-\frac{n^{3}}{2} \operatorname{Pr}\left[F_{2}\right]-\frac{n^{4}}{8} \operatorname{Pr}\left[F_{3}\right] .
$$

We shall prove this claim by the Palm theory and Boole's inequalities. For clarity, we use $X_{1}^{\prime}, X_{2}^{\prime}, X_{3}^{\prime}$, and $X_{4}^{\prime}$ to denote elements of $\mathcal{P}_{n}$. For any $\left\{x_{1}, x_{2}, x_{3}\right\} \subseteq V$, let

$$
\begin{aligned}
h_{2}\left(\left\{x_{1}, x_{2}, x_{3}\right\}, V\right) & =h_{1}\left(\left\{x_{1}, x_{2}\right\}, V\right) \cdot h_{1}\left(\left\{x_{1}, x_{3}\right\}, V\right) \\
& +h_{1}\left(\left\{x_{2}, x_{1}\right\}, V\right) \cdot h_{1}\left(\left\{x_{2}, x_{3}\right\}, V\right) \\
& +h_{1}\left(\left\{x_{3}, x_{1}\right\}, V\right) \cdot h_{1}\left(\left\{x_{3}, x_{2}\right\}, V\right) .
\end{aligned}
$$

For any $\left\{x_{1}, x_{2}, x_{3}, x_{4}\right\} \subseteq V$, let

$$
\begin{aligned}
h_{3}\left(\left\{x_{1}, x_{2}, x_{3}, x_{4}\right\}, V\right) & =h_{1}\left(\left\{x_{1}, x_{2}\right\}, V\right) \cdot h_{1}\left(\left\{x_{3}, x_{4}\right\}, V\right) \\
& +h_{1}\left(\left\{x_{1}, x_{3}\right\}, V\right) \cdot h_{1}\left(\left\{x_{2}, x_{4}\right\}, V\right) \\
& +h_{1}\left(\left\{x_{1}, x_{4}\right\}, V\right) \cdot h_{1}\left(\left\{x_{2}, x_{3}\right\}, V\right) .
\end{aligned}
$$

Let $F_{1}^{\prime}\left(\left\{X_{1}^{\prime}, X_{2}^{\prime}\right\}\right)$ be the event that

$$
h_{1}\left(\left\{X_{1}^{\prime}, X_{2}^{\prime}\right\}, \mathcal{P}_{n}\right)=1,
$$

$F_{2}^{\prime}\left(\left\{X_{1}^{\prime}, X_{2}^{\prime}, X_{3}^{\prime}\right\}\right)$ be the event that

$$
h_{2}\left(\left\{X_{1}^{\prime}, X_{2}^{\prime}, X_{3}^{\prime}\right\}, \mathcal{P}_{n}\right)=1,
$$


and $F_{3}^{\prime}\left(\left\{X_{1}^{\prime}, X_{2}^{\prime}, X_{3}^{\prime}, X_{4}^{\prime}\right\}\right)$ be the event that

$$
h_{3}\left(\left\{X_{1}^{\prime}, X_{2}^{\prime}, X_{3}^{\prime}, X_{4}^{\prime}\right\}, \mathcal{P}_{n}\right)=1
$$

According to the Palm theory (Theorem 9), we have

$$
\begin{aligned}
& \sum_{\left\{X_{1}^{\prime}, X_{2}^{\prime}\right\} \subseteq \mathcal{P}_{n}} \operatorname{Pr}\left[F_{1}^{\prime}\left(\left\{X_{1}^{\prime}, X_{2}^{\prime}\right\}\right)\right] \\
&= \mathbf{E}\left[\sum_{\left\{X_{1}^{\prime}, X_{2}^{\prime}\right\} \subseteq \mathcal{P}_{n}} h_{1}\left(\left\{X_{1}^{\prime}, X_{2}^{\prime}\right\}, \mathcal{P}_{n}\right)\right] \\
&= \frac{n^{2}}{2 !} \mathbf{E}\left[h_{1}\left(\left\{X_{1}, X_{2}\right\},\left\{X_{1}, X_{2}\right\} \cup \mathcal{P}_{n}\right)\right] \\
&= \frac{n^{2}}{2} \operatorname{Pr}\left[F_{1}\right], \\
&\left\{X_{1}^{\prime}, X_{2}^{\prime}, X_{3}^{\prime}\right\} \subseteq \mathcal{P}_{n} \operatorname{Pr}\left[F_{2}^{\prime}\left(\left\{X_{1}^{\prime}, X_{2}^{\prime}, X_{3}^{\prime}\right\}\right)\right] \\
&= \mathbf{E}\left[\sum_{\left\{X_{1}^{\prime}, X_{2}^{\prime}, X_{3}^{\prime}\right\} \subseteq \mathcal{P}_{n}} h_{2}\left(\left\{X_{1}^{\prime}, X_{2}^{\prime}, X_{3}^{\prime}\right\}, \mathcal{P}_{n}\right)\right] \\
&=\frac{n^{3}}{3 !} \mathbf{E}\left[h_{2}\left(\left\{X_{1}, X_{2}, X_{3}\right\},\left\{X_{1}, X_{2}, X_{3}\right\} \cup \mathcal{P}_{n}\right)\right] \\
&=3 \frac{n^{3}}{3 !} \operatorname{Pr}\left[F_{2}\right]=\frac{n^{3}}{2} \operatorname{Pr}\left[F_{2}\right],
\end{aligned}
$$

and

$$
\begin{aligned}
& \sum_{\left\{X_{1}^{\prime}, X_{2}^{\prime}, X_{3}^{\prime}, X_{4}^{\prime}\right\} \subseteq \mathcal{P}_{n}} \operatorname{Pr}\left[F_{3}^{\prime}\left(\left\{X_{1}^{\prime}, X_{2}^{\prime}, X_{3}^{\prime}, X_{4}^{\prime}\right\}\right)\right] \\
= & \mathbf{E}\left[\sum_{\left\{X_{1}^{\prime}, X_{2}^{\prime}, X_{3}^{\prime}, X_{4}^{\prime}\right\} \subseteq \mathcal{P}_{n}} h_{3}\left(\left\{X_{1}^{\prime}, X_{2}^{\prime}, X_{3}^{\prime}, X_{4}^{\prime}\right\}, \mathcal{P}_{n}\right)\right] \\
= & \frac{n^{3}}{3 !} \mathbf{E}\left[h_{3}\left(\left\{X_{1}, X_{2}, X_{3}, X_{4}\right\},\left\{X_{1}, X_{2}, X_{3}, X_{4}\right\} \cup \mathcal{P}_{n}\right)\right] \\
= & 3 \frac{n^{4}}{4 !} \operatorname{Pr}\left[F_{2}\right]=\frac{n^{4}}{8} \operatorname{Pr}\left[F_{3}\right] .
\end{aligned}
$$

Applying Boole's inequalities and (3), (4), and (5), we have

$$
\begin{aligned}
\operatorname{Pr}\left[E_{1}\right] & \geq \sum_{\left\{X_{1}^{\prime}, X_{2}^{\prime}\right\} \subseteq \mathcal{P}_{n}} \operatorname{Pr}\left[F_{1}^{\prime}\left(\left\{X_{1}^{\prime}, X_{2}^{\prime}\right\}\right)\right] \\
& -\sum_{\left\{X_{1}^{\prime}, X_{2}^{\prime}, X_{3}^{\prime}\right\} \subseteq \mathcal{P}_{n}} \operatorname{Pr}\left[F_{2}^{\prime}\left(\left\{X_{1}^{\prime}, X_{2}^{\prime}, X_{3}^{\prime}\right\}\right)\right] \\
& -\sum_{\left\{X_{1}^{\prime}, X_{2}^{\prime}, X_{3}^{\prime}, X_{4}^{\prime}\right\} \subseteq \mathcal{P}_{n}} \operatorname{Pr}\left[F_{3}^{\prime}\left(\left\{X_{1}^{\prime}, X_{2}^{\prime}, X_{3}^{\prime}, X_{4}^{\prime}\right\}\right)\right] \\
& =\frac{n^{2}}{2} \operatorname{Pr}\left[F_{1}\right]-\frac{n^{3}}{2} \operatorname{Pr}\left[F_{2}\right]-\frac{n^{4}}{8} \operatorname{Pr}\left[F_{3}\right] .
\end{aligned}
$$

Then,

$$
\begin{aligned}
\operatorname{Pr}\left[F_{2}\right] & \leq 16 \int_{z_{1} \in A} \int_{r_{1}=\frac{R_{1}}{2}}^{\frac{R_{2}}{2}} \int_{z_{2} \in A} e^{-n\left(\pi r_{1}^{2}+c R_{2}\left\|z_{1}-z_{2}\right\|\right)} 2 \pi r_{1} d r_{1} d z_{1} d z_{2} \\
& \leq 16 \int_{z_{1} \in A} \int_{r_{1}=\frac{R_{1}}{2}}^{\frac{R_{2}}{2}} e^{-n \pi r_{1}^{2}} 2 \pi r_{1} d r_{1} d z_{1} \int_{z_{2} \in A} e^{-c n R_{2}\left\|z_{1}-z_{2}\right\|} d z_{2} \\
& \leq 16 \int_{z_{1} \in A} \int_{r_{1}=\frac{R_{1}}{2}}^{\frac{R_{2}}{2}} e^{-n \pi r_{1}^{2}} d\left(\pi r_{1}^{2}\right) d z_{1} \int_{\rho=0}^{\infty} e^{-c n R_{2} \rho} 2 \pi \rho d \rho \\
& =-\left(\left.\frac{16}{n} e^{-n \pi r^{2}}\right|_{r=\frac{R_{1}}{2}} ^{\frac{R_{2}}{2}}\right)|A| \cdot \frac{2 \pi}{\left(c n R_{2}\right)^{2}} \\
& =\frac{8 \pi}{c^{2}\left(n R_{2}^{2}\right) n^{3}}\left(n^{\frac{-\beta_{1}}{4}}-n^{-\frac{\beta_{2}}{4}}\right) \ln n .
\end{aligned}
$$

Let $z=\frac{x_{1}+x_{2}}{2}$ and $r=\frac{1}{2}\left\|x_{1}-x_{2}\right\|$. Then,

$$
\begin{aligned}
\operatorname{Pr}\left[F_{1}\right] & =\int_{z \in A} \int_{r=\frac{R_{1}}{2}}^{\frac{R_{2}}{2}} e^{-n \pi r^{2}} 8 \pi r d r d z=4 \int_{z \in A} \int_{r=\frac{R_{1}}{2}}^{\frac{R_{2}}{2}} e^{-n \pi r^{2}} d\left(\pi r^{2}\right) d z \\
& =-\left(\left.\frac{4}{n} e^{-n \pi r^{2}}\right|_{r=\frac{R_{1}}{2}} ^{\frac{R_{2}}{2}}\right)|A|=\frac{1}{n^{2}}\left(n^{-\frac{\beta_{1}}{4}}-n^{-\frac{\beta_{2}}{4}}\right) \ln n .
\end{aligned}
$$

Let $S_{2}$ denote the set

$\left\{\left(x_{1}, x_{2}, x_{3}\right) \mid \begin{array}{l}\frac{x_{1}+x_{2}}{2} \in A ; R_{1} \leq\left\|x_{1}-x_{2}\right\| \leq R_{2} ; x_{1}, x_{2} \notin D_{x_{1} x_{3}} ; \\ \frac{x_{1}+x_{3}}{2} \in A ; R_{1} \leq\left\|x_{1}-x_{3}\right\| \leq R_{2} ; x_{1}, x_{3} \notin D_{x_{1} x_{2}}\end{array}\right\}$.

Applying Lemma 5, if $\left(x_{1}, x_{2}, x_{3}\right) \in S_{2}$, we have

$$
\begin{aligned}
& \operatorname{Pr}\left[F_{2} \mid X_{1}=x_{1}, X_{2}=x_{2}, X_{3}=x_{3}\right] \leq e^{-n\left|D_{x_{1} x_{2}} \cup D_{x_{1} x_{3}}\right|} \\
& \leq e^{-n\left(\pi\left(\frac{1}{2}\left\|x_{1}-x_{2}\right\|\right)^{2}+c R_{2}\left\|\frac{x_{1}+x_{2}}{2}-\frac{x_{1}+x_{3}}{2}\right\|\right)} .
\end{aligned}
$$

Therefore,

$$
\begin{aligned}
\operatorname{Pr}\left[F_{2}\right] & =\iiint_{S_{2}} \operatorname{Pr}\left[F_{2} \mid X_{1}=x_{1}, X_{2}=x_{2}, X_{3}=x_{3}\right] d x_{1} d x_{2} d x_{3} \\
& \leq \iiint_{S_{2}} e^{-n\left(\pi\left(\frac{1}{2}\left\|x_{1}-x_{2}\right\|\right)^{2}+c R_{2}\left\|\frac{x_{1}+x_{2}}{2}-\frac{x_{1}+x_{3}}{2}\right\|\right)} d x_{1} d x_{2} d x_{3} .
\end{aligned}
$$

Let $z_{1}=\frac{x_{1}+x_{2}}{2}, r_{1}=\frac{1}{2}\left\|x_{1}-x_{2}\right\|, z_{2}=\frac{x_{1}+x_{3}}{2}$, and $\rho=\left\|z_{1}-z_{2}\right\|$.

Hence, our claim is true.
Let $S_{1}$ denote the set

$$
\left\{\left(x_{1}, x_{2}\right) \mid \frac{1}{2}\left(x_{1}+x_{2}\right) \in A, R_{1} \leq\left\|x_{1}-x_{2}\right\| \leq R_{2}\right\} \text {. }
$$

$\left\{\begin{array}{l|l}\left(x_{1}, x_{2}, x_{3}, x_{4}\right) & \begin{array}{l}\frac{x_{1}+x_{2}}{2} \in A ; R_{1} \leq\left\|x_{1}-x_{2}\right\| \leq R_{2} ; x_{1}, x_{2} \notin D_{x_{3} x_{4}} ; \\ \frac{x_{3}+x_{4}}{2} \in A ; R_{1} \leq\left\|x_{3}-x_{4}\right\| \leq R_{2} ; x_{3}, x_{4} \notin D_{x_{1} x_{2}}\end{array}\end{array}\right\}$. 
Applying Lemma 5, if $\left(x_{1}, x_{2}, x_{3}, x_{4}\right) \in S_{3}$, we have

$$
\begin{aligned}
& \operatorname{Pr}\left[F_{3} \mid X_{1}=x_{1}, X_{2}=x_{2}, X_{3}=x_{3}, X_{4}=x_{4}\right] \\
& \leq e^{-n \| D_{x_{1} x_{2}} \cup D_{x_{3} x_{4}} \mid} \leq e^{-n\left(\pi\left(\frac{1}{2}\left\|\mid x_{1}-x_{2}\right\|\right)^{2}+c R_{2}\left\|\frac{x_{1}+x_{2}}{2}-\frac{x_{3}+x_{4}}{2}\right\|\right) .}
\end{aligned}
$$

Therefore,

$$
\begin{aligned}
\operatorname{Pr}\left[F_{3}\right] & =\iiint \int_{S_{3}} \operatorname{Pr}\left[F_{3}\left[\begin{array}{l}
X_{1}=x_{1} \\
X_{2}=x_{2} \\
X_{3}=x_{3} \\
x_{4}=x_{4}
\end{array}\right] d x_{1} d x_{2} d x_{3} d x_{4}\right. \\
& \leq \iiint \int_{S_{3}} e^{-n\left(\pi\left(\frac{1}{2}\left\|x_{1}-x_{2}\right\|\right)^{2}+c R_{2}\left\|\frac{x_{1}+x_{2}}{2}-\frac{x_{3}+x_{4}}{2}\right\|\right)} \\
& d x_{1} d x_{2} d x_{3} d x_{4} .
\end{aligned}
$$

Let $z_{1}=\frac{x_{1}+x_{2}}{2}, r_{1}=\frac{1}{2}\left\|x_{1}-x_{2}\right\|, z_{2}=\frac{x_{3}+x_{4}}{2}, r_{2}=\frac{1}{2}\left\|x_{3}-x_{4}\right\|$, and $\rho=\left\|z_{1}-z_{2}\right\|$. Then,

$$
\begin{aligned}
\operatorname{Pr}\left[F_{3}\right] \leq & \int_{z_{1} \in A} \int_{r_{1}=\frac{R_{1}}{2}}^{\frac{R_{2}}{2}} \int_{z_{2} \in A} \int_{r_{2}=\frac{R_{1}}{2}}^{\frac{R_{2}}{2}} e^{-n\left(\pi r_{1}^{2}+c R_{2}\left\|z_{1}-z_{2}\right\|\right)} \\
& \left(8 \pi r_{1} d r_{1} d z_{1}\right)\left(8 \pi r_{2} d r_{2} d z_{2}\right) \\
\leq & \left(4 \int_{z_{1} \in A} \int_{r_{1}=\frac{R_{1}}{2}}^{\frac{R_{2}}{2}} e^{-n \pi r_{1}^{2}} 2 \pi d r_{1} d z_{1}\right) \\
& \left(8 \pi \frac{R_{2}}{2}\left(\frac{R_{2}}{2}-\frac{R_{1}}{2}\right) \int_{z_{2} \in A} e^{-c n R_{2}\left\|z_{1}-z_{2}\right\|} d z_{2}\right) \\
\leq & \left(4 \int_{z_{1} \in A} \int_{r_{1}=\frac{R_{1}}{2}}^{\frac{R_{2}}{2}} e^{-n \pi r_{1}^{2}} d\left(\pi r_{1}^{2}\right) d z_{1}\right) \\
& \left(8 \pi \frac{R_{2}}{2}\left(\frac{R_{2}}{2}-\frac{R_{1}}{2}\right) \int_{\rho=0}^{\infty} e^{-c n R_{2} \rho} 2 \pi \rho d \rho\right) \\
= & \left(\frac{\ln n}{n^{2}}\left(n^{-\frac{\beta_{1}}{4}}-n^{-\frac{\beta_{2}}{4}}\right)\right)\left(\frac{4 \pi^{2}}{\left(c n R_{2}\right)^{2}} R_{2}\left(R_{2}-R_{1}\right)\right) \\
= & \frac{4 \pi^{2}}{c^{2} n^{4}}\left(1-\frac{R_{1}}{R_{2}}\right)\left(n^{-\frac{\beta_{1}}{4}}-n^{-\frac{\beta_{2}}{4}}\right) \ln n .
\end{aligned}
$$

Combining (2), (6), (7), and (8), we have

$$
\begin{aligned}
\operatorname{Pr}\left[E_{1}\right] & \geq\left(\frac{1}{2}-\frac{4 \pi}{c^{2}\left(n R_{2}^{2}\right)}-\frac{\pi^{2}}{2 c^{2}}\left(1-\frac{R_{1}}{R_{2}}\right)\right)\left(n^{-\frac{\beta_{1}}{4}}-n^{-\frac{\beta_{2}}{4}}\right) \ln n \\
& \sim \frac{1}{2}\left(1-\frac{\pi^{2}}{c^{2}}\left(1-\frac{R_{1}}{R_{2}}\right)\right)\left(n^{-\frac{\beta_{1}}{4}}-n^{-\frac{\beta_{2}}{4}}\right) \ln n .
\end{aligned}
$$

Since $\frac{\pi^{2}}{c^{2}}\left(1-\frac{R_{1}}{R_{2}}\right)<1$ and $I_{n}=\Omega\left(\frac{\ln n}{n}\right)$, we have

$$
\operatorname{Pr}\left[E_{1}\right]=\Omega\left(\left(n^{-\frac{\beta_{1}}{4}}-n^{-\frac{\beta_{2}}{4}}\right) \ln n\right)
$$

and

$$
I_{n} \operatorname{Pr}\left[E_{1}\right]=\Omega\left(n^{1-\frac{\beta_{1}}{4}}\right) \rightarrow \infty .
$$

This complete the proof of Lemma 16.

\subsection{Upper Bounds for the Longest Edge Length}

In this section, we are going to give upper bounds for the length of the longest Gabriel edge.
Lemma 17. For any constant $\beta>2$, we have

$$
\lim _{n \rightarrow \infty} \operatorname{Pr}\left[\lambda\left(G\left(\mathcal{P}_{n}\right)\right) \geq \beta \sqrt{\frac{\ln n}{\pi n}}\right]=0 .
$$

Proof. Let $d=\beta \sqrt{\frac{\ln n}{\pi n}}$ and $r=\frac{d}{2}$. Pick a constant $\beta_{1} \in(2, \beta)$, and let $d^{\prime}=\beta_{1} \sqrt{\frac{\ln n}{\pi n}}, r^{\prime}=\frac{d^{\prime}}{2}$ and $\varepsilon=\frac{\left(r-r^{\prime}\right)}{\sqrt{2}}$. Let $\mathbf{C}_{r}$ be the collection of all feasible $r$-disks, whose diameters are contained in $\mathbb{D}$. If $x y$ is a Gabriel of the GG, there is no node on $D_{x y}$. Therefore, $\lambda\left(G\left(\mathcal{P}_{n}\right)\right) \geq d$ implies that there exists a disk of $\mathbf{C}_{r}$ on which there is no node of $\mathcal{P}_{n}$. Therefore,

$$
\operatorname{Pr}\left[\lambda\left(G\left(\mathcal{P}_{n}\right)\right) \geq d\right] \leq \operatorname{Pr}\left[\min _{C \in \mathbf{C}_{r}}\left|C \cap \mathcal{P}_{n}\right|=0\right] .
$$

Divide the plane into an $\varepsilon$-tessellation. The distance of any two points within a grid cell is at most $\left(r-r^{\prime}\right)$. If $A$ and $B$, respectively, are $r$-disk and $r^{\prime}$-disk with the same center, the grid cells intersected with $B$ are contained in $A$ since any point of $B$ is apart from $\partial A$ by at least $\left(r-r^{\prime}\right)$. So, the polyquadrate induced by $B$ is contained in $A$. Let $\left\{P_{1}, \cdots, P_{I_{n}}\right\}$ denote the set of polyquadrates induced by $r^{\prime}$-disks of $\mathbf{C}_{r^{\prime}}$, and $Y_{i}$ denote the number of nodes of $\mathcal{P}_{n}$ on $P_{i}$. Since any $r$-disk of $\mathbf{C}_{r}$ contains at least one $P_{i}$, we have

$$
\begin{aligned}
\operatorname{Pr}\left[\min _{C \in \mathbf{C}_{r}}\left|C \cap \mathcal{P}_{n}\right|=0\right] & \leq \operatorname{Pr}\left[\min _{i=1}^{I_{n}} Y_{i}=0\right] \\
& =1-\operatorname{Pr}\left[\min _{i=1}^{I_{n}} Y_{i}>0\right] .
\end{aligned}
$$

Note that $Y_{i}$ is a Poisson RV with rate $n\left|P_{i}\right|$. Assume that $E$ is the collection of polyquadrates induced by $r^{\prime}$-disks with center on $\mathbb{D}_{r^{\prime}}(0)$ and $F$ is the collection of polyquadrates induced by feasible $r^{\prime}$-disks with their center on $\mathbb{D} \backslash \mathbb{D}_{r^{\prime}}(0)$. Since $E \cup F=\left\{P_{1}, \cdots, P_{I_{n}}\right\}$,

$$
\begin{aligned}
\operatorname{Pr}\left[\min _{i=1}^{I_{n}} Y_{i}=0\right] & =\operatorname{Pr}\left[\min _{P_{i} \in E} Y_{i}=0 \text { or } \min _{P_{i} \in F} Y_{i}=0\right] \\
& \leq \operatorname{Pr}\left[\min _{P_{i} \in E} Y_{i}=0\right]+\operatorname{Pr}\left[\min _{P_{i} \in F} Y_{i}=0\right] .
\end{aligned}
$$

For any $P_{i} \in E$, we have

$$
n\left|P_{i}\right| \geq n \frac{1}{4} \pi\left(2 r^{\prime}\right)^{2}=\frac{1}{4} \beta_{1}^{2} \ln n>\ln n .
$$

Applying Lemma 7, we also have $|E|=\Theta\left(\frac{1}{\varepsilon^{2}}\right)=\Theta\left(\frac{n}{\ln n}\right)$. Therefore, by Lemma 8 ,

$$
\operatorname{Pr}\left[\min _{P_{i} \in E} Y_{i}=0\right]=1-\operatorname{Pr}\left[\min _{P_{i} \in E} Y_{i}>0\right] \sim 0 .
$$

For any $P_{i} \in F$, we have

$$
n\left|P_{i}\right| \geq \frac{1}{2} n\left(\frac{1}{4} \pi\left(2 r^{\prime}\right)^{2}\right)>\frac{1}{2} \ln n .
$$

Applying Lemma 7, we also have $|F|=\Theta\left(\frac{r^{\prime}}{\varepsilon^{2}}\right)=\Theta$ $\left(\sqrt{\frac{n}{\ln n}}\right)$. Therefore, by Lemma 8 , 


$$
\operatorname{Pr}\left[\min _{P_{i} \in F} Y_{i}=0\right]=1-\operatorname{Pr}\left[\min _{P_{i} \in F} Y_{i}>0\right] \sim 0 .
$$

Put all together, and the lemma is proved.

Lemmas 16 and 17, respectively, give lower and upper bounds for the length of the longest Gabriel edge. Hence, Theorem 1 is now an immediate consequence of Lemmas 16 and 17 .

\section{EXPected Number of Long Edges}

In the previous section, we proved that the ratio of the length of the longest Gabriel edge to $\sqrt{\frac{\ln n}{n \pi}}$ is a.a.s. equal to 2 . In this section, we are going to prove Theorem 2, which gives the expectation of the number of long Gabriel edges.

Proof of Theorem 2. Assume $Y$ and $V$ are point sets and $Y \subseteq V$. Let $h_{r}(Y, V)$ denote a function such that $h_{r}(Y=$ $\left.\left\{x_{1}, x_{2}\right\}, V\right)=1$ only if $\left\|x_{1}-x_{2}\right\| \geq r$ and there is no other node of $V$ in the disk area $D_{x_{1} x_{2}}$; otherwise, $h_{r}(Y, V)=0$. Let $X_{1}$ and $X_{2}$ denote independent random points with uniform distribution over $\mathrm{D}$ and independent of $\mathcal{P}_{n}$. According to the Palm theory,

$$
\begin{aligned}
\mathbf{E}\left[N\left(G\left(\mathcal{P}_{n}\right), r\right)\right] & =\mathbf{E}\left[\sum_{\left\{X_{1}^{\prime}, X_{2}^{\prime}\right\} \subseteq \mathcal{P}_{n}} h_{r}\left(\left\{X_{1}^{\prime}, X_{2}^{\prime}\right\}, \mathcal{P}_{n}\right)\right] \\
& =\frac{n^{2}}{2 !} \mathbf{E}\left[h_{r}\left(\left\{X_{1}, X_{2}\right\},\left\{X_{1}, X_{2}\right\} \cup \mathcal{P}_{n}\right)\right] .
\end{aligned}
$$

Let $F(r)$ be the probability of the event that $X_{1} X_{2}$ is a Gabriel edge and $\left\|X_{1}-X_{2}\right\| \geq r$. Then,

$$
F(r)=\mathbf{E}\left[h_{r}\left(\left\{X_{1}, X_{2}\left\{X_{1}, X_{2}\right\} \cup \mathcal{P}_{n}\right)\right]\right.
$$

$$
\text { and } \begin{aligned}
& F(r)=\iint_{\substack{x_{1}, x_{2} \in \mathbb{D} \\
\left\|x_{1}-x_{2}\right\| \geq r}} \\
& \operatorname{Pr}\left[X_{a} X_{2} \text { is a Gabriel edge| } \begin{array}{l}
X_{1}=x_{1} \\
X_{2}=x_{2}
\end{array}\right] d x_{1} d x_{2} \\
= & \iint_{\substack{x_{1}, x_{2} \in \mathbb{D} \\
\left\|x_{2}-x_{2}\right\| \geq r}} e^{-n v_{\left\|x_{1}-x_{2}\right\| / 2}\left(\frac{x_{1}+x_{2}}{2}\right)} d x_{1} d x_{2}, \\
= & \int_{\rho=\frac{r}{2}}^{\infty} \int_{z \in \mathbb{D})} e^{-n v_{\rho}(z)} 4 r \theta(z, \rho) d z d r .
\end{aligned}
$$

In the last equality, we let $z=\frac{x_{1}+x_{2}}{2}$ and $\rho=\frac{1}{2}\left\|x_{1}-x_{2}\right\|$. According to Theorem 1,

$$
\begin{aligned}
F\left(2 \sqrt{\frac{\ln n+\xi}{n \pi}}\right) & \sim F\left(2 \sqrt{\frac{\ln n+\xi}{n \pi}}\right)-F\left(3 \sqrt{\frac{\ln n}{n \pi}}\right) \\
& =\int_{\rho=\sqrt{\frac{\ln n+\xi}{n \pi}}}^{\frac{3}{2} \sqrt{\frac{\ln n}{n \pi}}} \int_{z \in \mathbb{D} D} e^{-n v_{\rho}(z)} 4 r \theta(z, \rho) d z d \rho .
\end{aligned}
$$

Hence, applying Lemma 11,

$$
\mathbf{E}\left[N\left(G\left(\mathcal{P}_{n}\right), 2 \sqrt{\frac{\ln n+\xi}{n \pi}}\right)\right]=\frac{n^{2}}{2 !} F\left(2 \sqrt{\frac{\ln n+\xi}{n \pi}}\right) \sim 2 e^{-\xi} .
$$

\section{Asymptotic Probability Distribution of the MAXIMAL LENGTH}

Let $R_{n}=2 \sqrt{\frac{\ln n+\xi_{n}}{\pi n}}$ for some sequence $\xi_{n}$ such that $\xi_{n} \rightarrow \infty$, $\xi_{n}=o(\ln n)$, and $m(n)=n(n-1) / 2$. For applying Theorem 10 (Brun's sieve) to prove Theorem 3 , let $B_{i}$ be the event that the edge between the $i$ th pair of nodes is a Gabriel edge whose length is at least $r_{\xi}$ but less than $R_{n}$ for $1 \leq i \leq m(\operatorname{Po}(n))$, and $Y$ be the number of $B_{i}$ that holds. Then, $Y$ is exactly the number of Gabriel edges whose lengths are at least $r_{\xi}=2 \sqrt{\frac{\ln n+\xi}{\pi n}}$ but less than $R_{n}$. According to Theorem 2, $Y$ is a.a.s. equal to the number of Gabriel edges whose length is at least $r_{\xi}$.

For any fixed integer $k$, let $K_{1}$ denote the collection of set $\left\{i_{1}, \cdots, i_{k}\right\} \subseteq\{1, \cdots, m(P o(n))\}$ such that $e_{i_{1}}, \cdots, e_{i_{k}}$ are not incident to the same nodes, and $K_{2}$ be the collection of set $\left\{i_{1}, \cdots, i_{k}\right\} \subseteq\{1, \cdots, m(\operatorname{Po}(n))\}$ such that $e_{i_{1}}, \cdots, e_{i_{k}}$ have some common endpoints. Then,

$$
\begin{aligned}
S^{(k)}= & \sum_{\left\{i_{1}, \cdots, i_{k}\right\} \subseteq\{1, \cdots, m(\operatorname{Po}(n))\}} \operatorname{Pr}\left[B_{i_{1}} \wedge \cdots \wedge B_{i_{k}}\right] \\
= & \sum_{\left\{i_{1}, \cdots, i_{k}\right\} \in K_{1}} \operatorname{Pr}\left[B_{i_{1}} \wedge \cdots \wedge B_{i_{k}}\right] \\
& +\sum_{\left\{i_{1}, \cdots, i_{k}\right\} \in K_{2}} \operatorname{Pr}\left[B_{i_{1}} \wedge \cdots \wedge B_{i_{k}}\right] .
\end{aligned}
$$

In Lemma 18, we shall prove that the expectation of the sum over $\left\{i_{1}, \cdots, i_{k}\right\} \in K_{1}$ are asymptotically equal to $\left(2 e^{-\xi}\right)^{k}$. In Lemma 19, we shall prove that the expectation of the sum over $\left\{i_{1}, \cdots, i_{k}\right\} \in K_{2}$ are asymptotically equal to zero. Therefore, Theorem 3 follows Lemmas 18 and 19 by applying Theorem 10 .

In the proofs of Lemmas 18 and 19, for applying the Palm theory, let $\mathcal{X}_{l}=\left\{X_{1}, \cdots, X_{l}\right\}$ denote a uniform $l$-points process over $\mathbb{D}$ and independent of $\mathcal{P}_{n}$, and $\mathbf{B}_{i}$ be the event that the edge between the $i$ th pair of nodes of $\mathcal{X}_{l}$ is a Gabriel edge over $\mathcal{X}_{l} \cup \mathcal{P}_{n}$ whose length is at least $r_{\xi}$ but less than $R_{n}$ for $1 \leq i \leq m(l)$. In addition, let $e_{i}$ denote the edge of $X_{2 i-1} X_{2 i}$ (or $x_{2 i-1} x_{2 i}$ ), $z_{i}=\frac{x_{2 i-1}+x_{2 i}}{2}$ be the midpoint of edge $e_{i}$, and $r_{i}=\left\|\frac{x_{2 i-1}-x_{2 i}}{2}\right\|$ be the radius of $D_{x_{2 i-1} x_{2 i}} .\left\|e_{i}\right\|$ is shorthand for $\left\|x_{2 i-1}-x_{2 i}\right\|$, i.e., the length of edge $e_{i}$.

Lemma 18. For any fixed $k$,

$$
\mathbf{E}\left[\sum_{\left\{i_{1}, \cdots, i_{k}\right\} \in K_{1}} \operatorname{Pr}\left[B_{i_{1}} \wedge \cdots \wedge B_{i_{k}}\right]\right] \sim\left(2 e^{-\xi}\right)^{k} .
$$

Proof. Applying the Palm theory and due to the identity property, we have

$$
\begin{aligned}
& \mathbf{E}\left[\sum_{\left\{i_{1}, \cdots, i_{k}\right\} \in K_{1}} \operatorname{Pr}\left[B_{i_{1}} \wedge \cdots \wedge B_{i_{k}}\right]\right] \\
& =\frac{n^{2 k}}{(2 k) !}\left(\frac{\left(\begin{array}{c}
2 k \\
2
\end{array}\right)\left(\begin{array}{c}
2 k-2 \\
2
\end{array}\right) \cdots\left(\begin{array}{c}
2 \\
2
\end{array}\right)}{k !} \operatorname{Pr}\left[\mathbf{B}_{1} \wedge \cdots \wedge \mathbf{B}_{k}\right]\right) \\
& =\frac{1}{k !}\left(\frac{n^{2}}{2}\right)^{k} \operatorname{Pr}\left[\mathbf{B}_{1} \wedge \cdots \wedge \mathbf{B}_{k}\right] .
\end{aligned}
$$


It is sufficient to show that, for any fixed $k$,

$$
\left(\frac{n^{2}}{2}\right)^{k} \operatorname{Pr}\left[\mathbf{B}_{1} \wedge \cdots \wedge \mathbf{B}_{k}\right] \sim\left(2 e^{-\xi}\right)^{k}
$$

For $k=1$, we have

$$
\operatorname{Pr}\left[\mathbf{B}_{1} \mid X_{1}=x_{1}, X_{2}=x_{2}\right]=e^{-n \nu_{\left\|x_{1}-x_{2}\right\| / 2}\left(\frac{x_{1}+x_{2}}{2}\right)} .
$$

Hence,

$$
\begin{aligned}
& \frac{n^{2}}{2} \operatorname{Pr}\left[\mathbf{B}_{1}\right]=\frac{n^{2}}{2} \iint_{\substack{x_{\xi}, x_{2} \\
r_{\xi}\left\|x_{1}-x_{2}\right\| \leq R_{n}}} \operatorname{Pr}\left[\mathbf{B}_{1} \mid X_{1}=x_{1}, X_{2}=x_{2}\right] d x_{2} d x_{1} \\
& =\frac{n^{2}}{2} \iint_{\substack{x_{\xi} \leq\left\|x_{1} \in x_{2}\right\| x_{2} \| \leq R_{n}\\
}} e^{-n \nu_{\left\|x_{1}-x_{2}\right\| / 2}\left(\frac{x_{1}+x_{2}}{2}\right)} d x_{2} d x_{1} \\
& =\frac{n^{2}}{2} \int_{r_{1}=\frac{r_{\xi}}{2}}^{\frac{R_{n}}{2}} \int_{z_{1} \in \mathbb{D}} e^{-\nu_{r_{1}}\left(z_{1}\right)} 4 r_{1} \theta\left(z_{1}, r_{1}\right) d z_{1} d r_{1} 2 e^{-\xi} .
\end{aligned}
$$

The last asymptotic equality is given by Lemma 11 . So, the asymptotic equality (9) is true for $k=1$.

Now, fix $k \geq 2$. We have

$$
\begin{aligned}
& \left(\frac{n^{2}}{2}\right)^{k} \operatorname{Pr}\left[\mathbf{B}_{1} \wedge \cdots \wedge \mathbf{B}_{k}\right] \\
& =\left(\frac{n^{2}}{2}\right)^{k} \int_{\substack{x_{1}, \cdots, x_{2} \in \mathbb{D} \\
r_{\xi} \leq\left\|_{1}\right\| \leq R_{n}}} \operatorname{Pr}\left[\mathbf{B}_{1} \wedge \cdots \wedge \mathbf{B}_{k} \mid \begin{array}{c}
\text { for any } 1 \leq i \leq 2 k, \\
X_{i}=x_{i}
\end{array}\right] \\
& \left(\prod_{i=1}^{2 k} d x_{i}\right) .
\end{aligned}
$$

Let $z=\left(z_{1}, \cdots, z_{k}\right)$ and $r=\left(r_{1}, \cdots, r_{k}\right)$. We have

$\operatorname{Pr}\left[\mathbf{B}_{1} \wedge \cdots \wedge \mathbf{B}_{k} \mid\right.$ for any $\left.1 \leq i \leq 2 k, X_{i}=x_{i}\right] \leq e^{-n \nu_{r}(z)}$.

In addition, if $z \in C_{k k}\left(\frac{R_{n}}{2}\right)$,

$\operatorname{Pr}\left[\mathbf{B}_{1} \wedge \cdots \wedge \mathbf{B}_{k} \mid\right.$ for any $\left.1 \leq i \leq 2 k, X_{i}=x_{i}\right]=e^{-n \nu_{r}(z)}$.

From (10) and Lemmas 12 and 13,

$$
\begin{aligned}
& \left(\frac{n^{2}}{2}\right)^{k} \operatorname{Pr}\left[\mathbf{B}_{1} \wedge \cdots \wedge \mathbf{B}_{k} \text { and } z \in \mathbb{D}^{k} \backslash C_{k k}\left(\frac{R_{n}}{2}\right)\right] \\
& \leq\left(\frac{n^{2}}{2}\right)^{k} \int_{r \in\left[\frac{r_{2}}{2}, \frac{R_{n}}{2}\right]^{k}} \int_{z \in \mathbb{D}^{k} \backslash C_{k k}\left(\frac{R_{n}}{2}\right)} \mathbf{1}_{z}^{r} e^{-n \nu_{r}(z)} \prod_{i=1}^{k} 4 r_{i} \theta\left(z_{i}, r_{i}\right) d z_{i} d r_{i} \\
& =o(1) .
\end{aligned}
$$

From (11) and Lemma 14,

$$
\begin{aligned}
& \left(\frac{n^{2}}{2}\right)^{k} \operatorname{Pr}\left[\mathbf{B}_{1} \wedge \cdots \wedge \mathbf{B}_{k} \text { and } z \in C_{k k}\left(\frac{R_{n}}{2}\right)\right] \\
& =\left(\frac{n^{2}}{2}\right)^{k} \int_{r \in\left[\frac{\left.r_{\xi}, \frac{R_{n}}{2}\right]^{k}}{k}\right.} \int_{z \in C_{k k}\left(\frac{R_{n}}{2}\right)} e^{-n \nu_{r}(z)} \prod_{i=1}^{k} 4 r_{i} \theta\left(z_{i}, r_{i}\right) d z_{i} d r_{i} \\
& \sim\left(2 e^{-\xi}\right)^{k} .
\end{aligned}
$$

So, the asymptotic equality (9) is also true for any fixed $k \geq 2$. Hence, Lemma 18 is proved.

Lemma 19. For any fixed $k$,

$$
\mathbf{E}\left[\sum_{\left\{i_{1}, \cdots, i_{k}\right\} \in K_{2}} \operatorname{Pr}\left[B_{i_{1}} \wedge \cdots \wedge B_{i_{k}}\right]\right] \sim o(1) .
$$

Proof. For $3 \leq m \leq 2 k-1$, let $K_{2}^{m}$ be the collection of set $\left\{i_{1}, \cdots, i_{k}\right\} \in K_{2}$ such that $e_{i_{1}}, \cdots, e_{i_{k}}$ have some common endpoints and the total number of nodes incident to $e_{i_{1}}, \cdots, e_{i_{k}}$ is $m$. Then, $K_{2}=\bigcup_{m=3}^{2 k-1} K_{2}^{m}$ and

$$
\begin{aligned}
& \sum_{\left\{i_{1}, \cdots, i_{k}\right\} \in K_{2}} \operatorname{Pr}\left[B_{i_{1}} \wedge \cdots \wedge B_{i_{k}}\right] \\
= & \sum_{m=3}^{2 k-1} \sum_{\left\{i_{1}, \cdots, i_{k}\right\} \in K_{2}^{m}} \operatorname{Pr}\left[B_{i_{1}} \wedge \cdots \wedge B_{i_{k}}\right] .
\end{aligned}
$$

For any $\left\{i_{1}, \cdots, i_{k}\right\} \in K_{2}^{m}, e_{i_{1}}, \cdots, e_{i_{k}}$ contain a subgraph that is a forest incident to $m$ nodes and with at least one tree composed of more than two nodes. Let $\tau_{m}$ denote the collection of $k$-edges forest topologies that are incident to exactly $m$ nodes and contain at least one tree component composed of more than two nodes. Applying the Palm theory, we have

$$
\begin{gathered}
\mathbf{E}\left[\sum_{\left\{i_{1}, \cdots, i_{k}\right\} \in K_{2}} \operatorname{Pr}\left[B_{i_{1}} \wedge \cdots \wedge B_{i_{k}}\right]\right] \\
\leq \sum_{m=3}^{2 k-1} \sum_{\tau \in \tau_{m}} O\left(n^{m}\right) \operatorname{Pr}\left[\bigwedge_{e_{i} \in \tau} \mathbf{B}_{i}\right] .
\end{gathered}
$$

Note that, for a fixed $k$, we have $\left|\tau_{m}\right|=O(1)$. Therefore, it is enough to prove that, for any forest topology $\tau \in \tau_{m}$,

$$
n^{m} \operatorname{Pr}\left[\bigwedge_{e_{i} \in \tau} \mathbf{B}_{i}\right] \sim o(1) .
$$

Let $\tau=\left\{T_{1}, \cdots, T_{t}\right\}$ denote a forest composed of $e_{1}, \cdots, e_{m-t}$ incident to $X_{1}, \cdots, X_{m}$ such that at least one tree contains more than two nodes. Let $E_{\tau}$ be the event $B_{1} \wedge \cdots \wedge B_{m-t}$. Without loss of generality, we assume $e_{i}$ is contained in $T_{i}$ for $1 \leq i \leq t$. Let $\Pi_{i}$ denote the collection of all $i$-partition of $\{1,2, \cdots, t\}$. For any $\varpi=\left\{P_{1}, P_{2}, \cdots, P_{i}\right\}$ $\in \Pi_{i}$, let $m_{\varpi}(j)=\sum_{k \in P_{j}}\left|V\left(T_{k}\right)\right|, V_{\varpi}(j)=\bigcup_{k \in P_{j}} V\left(T_{k}\right), \tau_{\varpi}(j)$ $=\left\{T_{k}: k \in P_{j}\right\}$, and $S(\varpi)$ be the set of $z \in \mathbb{D}^{m-t}$ such that $\bigcup_{z_{i} \in V_{\varpi}(j)} B\left(z_{i}, \frac{R_{n}}{2}\right)$ forms a connected component.

For a fixed topology $\tau$, let

$S_{\tau}^{i j}=$

$\left\{x \in \mathbb{D}^{m} \mid \begin{array}{c}\text { for any } 1 \leq k \leq m-1, r_{\xi} \leq\left\|x_{k 1}-x_{k 2}\right\| \leq R_{n} \\ \text { and }\left\|z_{i}\right\| \geq\left\|z_{k}\right\| \geq\left\|z_{j}\right\|\end{array}\right\}$, $S^{i j}=$

$\left\{z \in C_{(m-t) 1}\left(\frac{R_{n}}{2}\right) \mid\left\|z_{i}\right\| \geq\left\|z_{j}\right\|\right.$ for any $\left.1 \leq j \leq m-t\right\}$.

First, consider $t=1$, i.e., $X_{1}, \cdots, X_{m}$ form a tree by $e_{1}, \cdots, e_{m-1}$. We have 

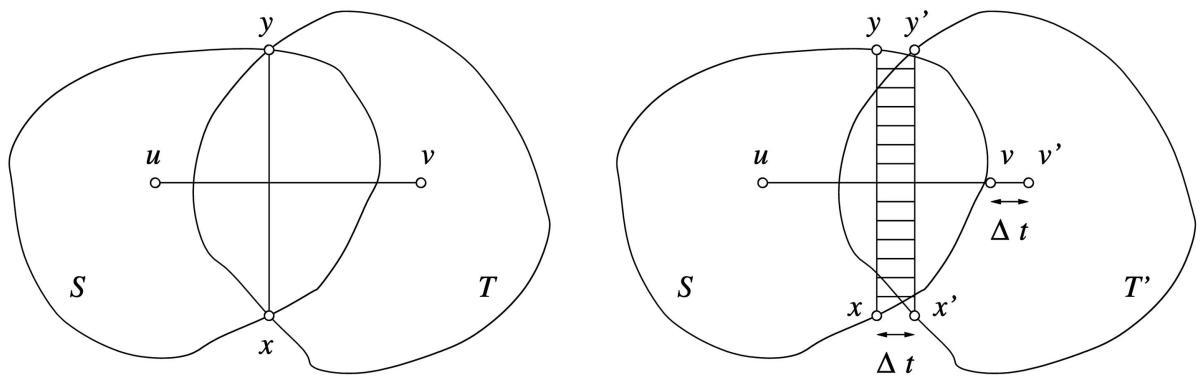

Fig. 5. Two intersecting domains.

$$
\begin{aligned}
& n^{m} \operatorname{Pr}\left[E_{\tau}\right] \leq \sum_{1 \leq i \neq j \leq m-1} n^{m} \int_{x \in S_{\tau}^{i j}} \operatorname{Pr}\left[E_{\tau} \mid \begin{array}{c}
\text { for } 1 \leq i \leq m \\
X_{i}=x_{i}
\end{array}\right] \prod_{i=1}^{m} d x_{i} \\
& \leq O(1) \sum_{1 \leq i \neq j \leq m-1} n^{m} \int_{r_{i}=\frac{r_{\xi}}{2}}^{\frac{R_{n}}{2}} \int_{z \in S^{i j}} e^{-n\left(\nu_{r_{i}}\left(z_{i}\right)+c R_{n}\left\|z_{i}-z_{j}\right\|\right)} r_{i} \theta\left(z_{i}, r_{i}\right) \\
& \\
& \left(\prod_{k=1}^{m-1} d z_{k}\right) d r_{i} \\
& \sim 0 .
\end{aligned}
$$

The last equality is given by Lemma 15. Now, we consider $t>1$. For $\operatorname{Pr}\left[E_{\tau} \wedge\left\{Z \in C_{(m-t) 1}\left(\frac{R_{n}}{2}\right)\right\}\right]$, we have

$$
\begin{aligned}
& n^{m} \operatorname{Pr}\left[E_{\tau} \wedge\left\{Z \in C_{(m-t) 1}\left(\frac{R_{n}}{2}\right)\right\}\right] \\
& \leq \sum_{1 \leq i \neq j \leq m-t} n^{m} \int_{x \in S_{\tau}^{i j}} \operatorname{Pr}\left[E_{\tau} \mid X_{i}=x_{i} \text { for } 1 \leq i \leq m\right] \prod_{i=1}^{m} d x_{i} \\
& \leq O(1) \sum_{1 \leq i \neq j \leq m-1} n^{m} \int_{r \in\left[\frac{\xi}{2}, \frac{R_{n}}{2}\right]^{t}} \int_{z \in S^{i j}} e^{-n\left(\nu_{r_{i}}\left(z_{i}\right)+c R_{n}\left\|z_{i}-z_{j}\right\|\right)} \\
& \\
& \left(\prod_{k=1}^{t} r_{k} \theta\left(z_{k}, r_{k}\right) d r_{k}\right)\left(\prod_{k=1}^{m-t} d z_{k}\right) \\
& \sim 0 .
\end{aligned}
$$

The last equality is given by Lemma 15. For $\operatorname{Pr}\left[E_{\tau} \wedge\right.$ $\left.\left\{Z \in S_{\varpi}\right\}\right]$, applying the same argument used in Lemma 13 , we have

$$
\begin{aligned}
& n^{m} \operatorname{Pr}\left(E_{\tau} \wedge\left\{Z \in S_{\varpi}\right\}\right) \\
& \leq O(1) \prod_{j=1}^{i}\left(n^{m_{\varpi}(j)} \operatorname{Pr}\left(E_{\tau_{\varpi}(j)}\right)\right) \sim o(1) .
\end{aligned}
$$

Putting it all together, we have

$$
\begin{aligned}
n^{m} \operatorname{Pr}\left[E_{\tau}\right] & =\sum_{i=1}^{t} \sum_{\varpi \in \Pi_{i}} n^{m} \operatorname{Pr}\left[E_{\tau} \wedge\left\{Z \in S_{\varpi}\right\}\right] \\
& \leq O(1) \sum_{i=1}^{t} \sum_{\varpi \in \Pi_{i}}\left(\prod_{j=1}^{i} n^{m_{\varpi}(j)} \operatorname{Pr}\left(E_{\tau_{\varpi}(j)}\right)\right) \sim o(1) .
\end{aligned}
$$

Therefore, the lemma is proved.

\section{Conclusion}

The Gabriel graph is one of the widely used geometric structures in topology control of wireless ad hoc networks and can be constructed by distributed and localized algorithms. If all nodes have the same transmission radii, the maximal length of Gabriel edges is the smallest transmission radius for constructing the GG by only 1-hop neighbor information. In this paper, we assume a wireless ad hoc network is represented by a Poisson point process with mean $n$ on a unit-area disk. We first showed that the ratio of the maximal length of Gabriel edges to $\sqrt{\frac{\ln n}{\pi n}}$ is a.a.s. equal to 2 . Next, we proved that, for any constant $\xi$, the expected number of long Gabriel edges, whose lengths are at least $2 \sqrt{\frac{\ln n+\xi}{\pi n}}$, is a.a.s. equal to $2 e^{-\xi}$. This implies that, if $\xi \rightarrow \infty$, it is a.a.s. that the maximal length is less than $2 \sqrt{\frac{\ln n+\xi}{\pi n}}$. Last, we proved that the number of long Gabriel edges is asymptotically Poisson with mean $2 e^{-\xi}$. Therefore, the probability of the event that the maximal length of Gabriel edges is less than $2 \sqrt{\frac{\ln n+\xi}{\pi n}}$ is asymptotically equal to $\exp \left(-2 e^{-\xi}\right)$.

\section{APPENDIX}

First, we give two technical lemmas. Assume $S$ and $T$ are two convex compact sets, and $\partial S$ and $\partial T$ intersect only at two points $x$ and $y$. Let $u$ and $v$ be two points to the different side of $x y$ such that $u v$ and $x y$ are perpendicular. $T^{\prime}$ is obtained by shifting $T$ away from $S$ along $u v$ by distance $\triangle t$. Then, we have the following lemma:

Lemma 20. If the boundaries of $S$ and $T$ at $x$ and $y$ can be expressed by continue functions along the axis parallel to uv, then

$$
\left|S \cup T^{\prime}\right|-|S \cup T|=(\|x-y\|+o(1)) \triangle t .
$$

Proof. For convenience, assume $v$ is on $T$. After shifting $T$ apart from $S$ by $\triangle t, x, y$, and $v$ on $T$ are moved to $x^{\prime}, y^{\prime}$ and $v^{\prime}$ respectively. See Fig. 5. Then, $\left|S \cup T^{\prime}\right|-|S \cup T|$ is equal to the area of the shaded region between $x y$ and $x^{\prime} y^{\prime}$. Since the boundaries can be expressed by continue functions, the height of the shaded region can be estimated by $\|x-y\|+o(1)$. The distance between $x y$ and $x^{\prime} y^{\prime}$ is equal to $\triangle t$. So, the area of the shaded region can be expressed by $(\|x-y\|+o(1)) \triangle t$.

Lemma 21. Assume $R$ is a constant, $\frac{1}{2} R \leq r_{2} \leq r_{1} \leq R$, and $\left\|x_{2}-x_{1}\right\| \geq \sqrt{r_{1}^{2}-r_{2}^{2}}$. Let $t=\left\|x_{2}-x_{1}\right\|$ and $f(t)=$ $\left|B\left(x_{2}, r_{2}\right) \backslash B\left(x_{1}, r_{1}\right)\right|$. Then,

$$
f(t) \geq 0.16 R t .
$$

Proof. Assume $r_{1}$ is fixed. If $t<r_{1}+r_{2}$, let $y_{1} y_{2}$ be the common chord of $\partial B\left(x_{1}, r_{1}\right)$ and $\partial B\left(x_{2}, r_{2}\right)$. See Fig. $6 a$. 


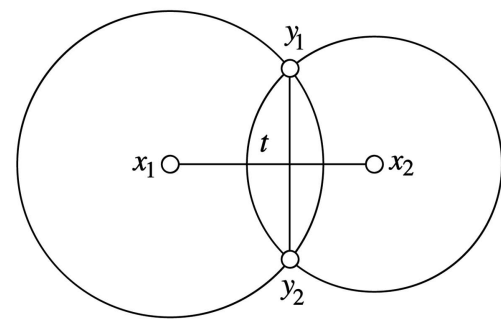

(a)

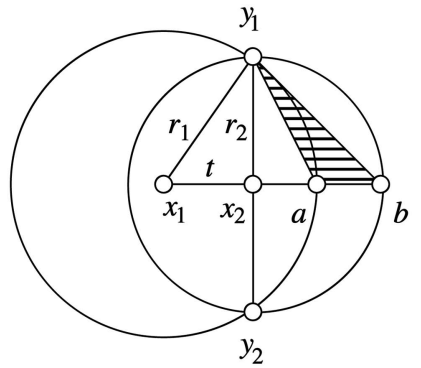

(b)

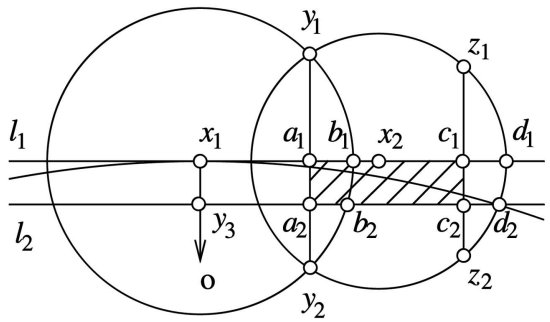

(c)

Fig. 6. The area of two intersecting disks.

Let $t_{0}=\sqrt{r_{1}^{2}-\left(\frac{1}{2} R\right)^{2}}$. For any $t \in\left[0, t_{0}\right]$, the minimum of $f(t)$ occurs as $r_{2}=\sqrt{r_{1}^{2}-t^{2}}$. So, without loss of generality, we assume $r_{2}=\sqrt{r_{1}^{2}-t^{2}}$. Let $a$ (respectively, $b$ ) be the intersection point of the ray $x_{1} x_{2}$ and circle $\partial B\left(x_{1}, r_{1}\right)$ (respectively, $\left.\partial B\left(x_{2}, r_{2}\right)\right)$. See Fig. 6b. Then,

$f(t) \geq 2\left|\triangle a b y_{1}\right|=2 \cdot \frac{1}{2}\left(r_{2}+t-r_{1}\right) r_{2}$

$\geq \frac{1}{2} R\left(\sqrt{r_{1}^{2}-t^{2}}-\left(r_{1}-t\right)\right)=\frac{1}{2} R \frac{\left(\sqrt{r_{1}^{2}-t^{2}}\right)^{2}-\left(r_{1}-t\right)^{2}}{\sqrt{r_{1}^{2}-t^{2}}+\left(r_{1}-t\right)}$

$=\frac{1}{2} R \frac{2 t\left(r_{1}-t\right)}{\sqrt{r_{1}^{2}-t^{2}}+\left(r_{1}-t\right)}=R t \frac{1}{\sqrt{\frac{r_{1}+t}{r_{1}-t}}+1} \geq \frac{1}{4+\sqrt{3}} R t>0.16 R t$.

Note that the maximum of $\sqrt{\frac{r_{1}+t}{r_{1}-t}}$ occurs as $r_{1}=R$, $r_{2}=\frac{1}{2} R$, and $t=\frac{\sqrt{3}}{2} R$. For any fixed $t \in\left[t_{0}, 2 R\right]$, the minimum of $f(t)$ occurs as $r_{2}=\frac{1}{2} R$. If $t<r_{1}+r_{2}$, according to Lemma 20, we have $f^{\prime}(t)=\left\|y_{1}-y_{2}\right\|$. If $t=t_{0}, y_{1} y_{2}$ is the diameter of $B\left(x_{2}, r_{2}\right)$. Therefore, for $t \in\left[t_{0}, 2 R\right], f^{\prime}(t)$ is decreasing, and $f(t)$ is concave. Since

$$
\frac{f(2 R)}{2 R}=\frac{\pi}{8} R>0.16 R=\frac{0.16 R t_{0}}{t_{0}},
$$

we have

$$
\frac{f(2 R)-0.16 R t_{0}}{2 R-t_{0}} \geq \frac{0.16 R t_{0}}{t_{0}}=0.16 R .
$$

Let $\triangle t=t-t_{0}$. Then,

$$
\begin{aligned}
f(t) & \geq 0.16 R t_{0}+\frac{f(2 R)-0.16 R t_{0}}{2 R-t_{0}} \triangle t \geq 0.16 R t_{0}+0.16 R \triangle t \\
& =0.16 R\left(t_{0}+\triangle t\right)=0.16 R t .
\end{aligned}
$$

Note that the final inequality does not depend on $r_{1}$. Therefore, the lemma is proved.

In the remainder of the Appendix, we give the proofs of Lemmas $5,11,12,13,14$, and 15.

Proof of Lemma 5. We prove the lemma by induction on $k$. We begin with $k=2$. Let $t=\left\|x_{2}-x_{1}\right\|$ and $f(t)=\mid B\left(x_{2}\right.$, $\left.r_{2}\right) \backslash B\left(x_{1}, r_{1}\right) \mid$. Since $\left|B\left(x_{2}, r_{2}\right) \backslash B\left(x_{1}, r_{1}\right)\right|>\mid B\left(x_{1}, r_{1}\right) \backslash$ $B\left(x_{2}, r_{2}\right) \mid$ if $r_{2}>r_{1}$, without loss of generality, we may assume $r_{1} \geq r_{2}$. According to Lemma 6, we have $f(t) \geq 0.16 R t$. If $r_{1}+r_{2} \leq t \leq 2 R$, since $B\left(x_{2}, r_{2}\right)$ and $B\left(x_{1}, r_{1}\right)$ are disjoint, we have $\nu_{r}(x)-\nu_{r_{1}}\left(x_{1}\right)=\nu_{r_{2}}\left(x_{2}\right) \geq \frac{1}{3} \pi\left(\frac{1}{2} R\right)^{2}=\frac{\pi}{24} R(2 R) \geq 0.03 R t$.

Now, we only need to consider $0 \leq t<r_{1}+r_{2}$. If $x_{1} \in \mathbb{D}_{r_{1}}(0)$, then $\nu_{r}\left(x_{1}, x_{2}\right)-\nu_{r_{1}}\left(x_{1}\right)$ is exactly $f(t)$ and, thus, the lemma follows immediately from $f(t) \geq 0.16 R t$. So, we assume that $x_{1} \notin \mathbb{D}(0)$. Note that, for the same distance $t, \nu_{r}\left(x_{1}, x_{2}\right)-\nu_{r_{1}}\left(x_{1}\right)$ achieves its minimum when both $x_{1}$ and $x_{2}$ are in $\partial \mathrm{DD}$. It is sufficient to prove the lemma for $x_{1}, x_{2} \in \partial \mathrm{DD}$. Let $\ell_{1}$ be the line perpendicular to $\mathbf{o} x_{1}$ and through $x_{1}$. Furthermore, if $x_{2}$ moves out of $\mathbb{D}, \nu_{r}(x)$ becomes smaller. So, consider that $x_{2}$ is on $\ell_{1}$. We use $d_{1}$ (respectively, $d_{2}$ ) to denote the intersection point of $\partial B\left(x_{2}, r_{2}\right)$ and $\ell_{1}$ (respectively, $\left.\partial \mathrm{ID}\right)$ far from $x_{1}$. Let $a_{1}$ be the intersection point of $\ell_{1}$ and $y_{1} y_{2}$, and $b_{1}$ be the intersection point of $\ell_{1}$ and $\partial B\left(x_{1}, r_{1}\right)$ near to $x_{2}$. We use $c_{1}$ to denote the point on $\ell_{1}$ and in $B\left(x_{2}, r_{2}\right)$ such that $\left\|c_{1}-d_{1}\right\|=\left\|a_{1}-b_{1}\right\|$. Let $z_{1}$ and $z_{2}$ be the two points in $\partial B\left(x_{2}, r_{2}\right)$ such that $z_{1} z_{2}$ is perpendicular to $\ell_{1}$ and through $c_{1}$. Let $\ell_{2}$ denote the line perpendicular to $\mathbf{o} x_{1}$ and through $d_{2}$. We use $y_{3}$ (respectively, $a_{2}$ and $c_{2}$ ) to denote the intersection point of $\ell_{2}$ and $\mathbf{o} x_{1}$ (respectively, $y_{1} y_{2}$ and $\left.z_{1} z_{2}\right)$, and $b_{2}$ to denote the intersection point of $\ell_{2}$ and $\partial B\left(x_{1}, r_{1}\right)$ near $x_{2}$. (See Fig. 6c.) We have

$$
\begin{aligned}
& \left\|a_{1}-c_{1}\right\|=\left\|a_{1}-x_{2}\right\|+\left\|x_{2}-c_{1}\right\| \leq\left\|a_{1}-x_{2}\right\| \\
& +\left\|x_{1}-a_{1}\right\|=t,
\end{aligned}
$$

and if $R \leq \frac{1}{5} R_{0}^{2}$,

$$
\begin{aligned}
\left\|a_{1}-a_{2}\right\| & =\left\|x_{1}-\mathbf{o}\right\|-\sqrt{\left\|d_{2}-\mathbf{o}\right\|^{2}-\left\|d_{2}-y_{3}\right\|^{2}} \\
& \leq R_{0}-\sqrt{R_{0}^{2}-(3 R)^{2}} \\
& =\frac{9 R^{2}}{R_{0}+\sqrt{R_{0}^{2}-(3 R)^{2}}} \leq \frac{9 R^{2}}{R_{0}+\frac{4}{5 R_{0}}}=\frac{5 R^{2}}{R_{0}} .
\end{aligned}
$$

The area of $a_{1} a_{2} b_{2} b_{1}$, surrounded by the segments $a_{1} a_{2}$, $a_{1} b_{1}, a_{2} b_{2}$, and arc $b_{1} b_{2}$, is larger than the area of $c_{1} c_{2} d_{2} d_{1}$, surrounded by the segments $c_{1} c_{2}, c_{1} d_{1}, c_{2} d_{2}$, and arc $d_{1} d_{2}$. Therefore, if $R \leq \frac{1}{100} R_{0}$, we have

$$
\begin{aligned}
\nu_{r}(x)-\nu_{r_{1}}\left(x_{1}\right) & \geq \frac{1}{2} f(t)-\left\|a_{1}-c_{2}\right\| \cdot\left\|a_{1}-a_{2}\right\| \\
& \geq 0.08 R t-\frac{5 R}{R_{0}} R t=0.03 R t
\end{aligned}
$$

and, thereby, the lemma for $k=2$ follows.

Next, we assume the lemma is true for at most $k-1$ nodes and we shall show that the lemma is true for $k$ nodes. If $k=3$, then 


$$
\begin{aligned}
\nu_{r}(x) & \geq \nu_{r_{1}}\left(x_{1}\right)+\nu_{r_{3}}\left(x_{3}\right) \geq \nu_{r_{1}}\left(x_{1}\right)+\frac{\pi r_{3}^{2}}{3} \geq \nu_{r_{1}}\left(x_{1}\right)+\frac{\pi}{12} R^{2} \\
& \geq \nu_{r_{1}}\left(x_{1}\right)+0.03 R \cdot 4 R \geq \nu_{r_{1}}\left(x_{1}\right)+0.03 R \sum_{i=1}^{2}\left\|x_{i+1}-x_{i}\right\| .
\end{aligned}
$$

If $k>3$, then by the induction hypothesis,

$$
\begin{aligned}
\nu_{r}(x) & \geq \nu_{\left(r_{1}, \cdots, r_{k-2}\right)}\left(x_{1}, \cdots, x_{k-2}\right)+\nu_{r_{k}}\left(x_{k}\right) \\
& \geq \nu_{r_{1}}\left(x_{1}\right)+0.03 R \sum_{i=1}^{k-3}\left\|x_{i+1}-x_{i}\right\|+\frac{\pi r_{k}^{2}}{3} \\
& \geq \nu_{r_{1}}\left(x_{1}\right)+0.03 R \sum_{i=1}^{k-3}\left\|x_{i+1}-x_{i}\right\|+0.03 R \cdot 4 R \\
& \geq \nu_{r_{1}}\left(x_{1}\right)+0.03 R \sum_{i=1}^{k-1}\left\|x_{i+1}-x_{i}\right\| .
\end{aligned}
$$

Therefore, the lemma is true by induction.

Proof of Lemma 11. First, we calculate the integration over $\mathrm{DD}_{r}(0)$.

$$
\begin{aligned}
& \frac{n^{2}}{2} \int_{r=\frac{r_{\xi}}{2}}^{\frac{R_{n}}{2}} \int_{z \in \mathbb{D}_{r}(0)} e^{-n \nu_{r}(z)} 4 r \theta(z, r) d z d r \\
& =\frac{n^{2}}{2} \int_{r=\frac{r_{\xi}}{2}}^{\frac{R_{n}}{2}} \int_{z \in \mathbb{D}_{r}(0)} e^{-n \pi r^{2}} 8 \pi r d z d r \\
& \sim 2 n^{2} \int_{r=\frac{r_{\xi}}{2}}^{\frac{R_{n}}{2}} e^{-n \pi r^{2}} 2 \pi r d r=2 n^{2} \int_{r=\frac{r_{\xi}}{2}}^{\frac{R_{n}}{2}} e^{-n \pi r^{2}} d\left(\pi r^{2}\right) \\
& =2 n^{2}\left(-\frac{1}{n} e^{-\left.n \pi r^{2}\right|_{r=\frac{R_{n}}{2}} ^{\frac{R_{n}}{2}}}\right) \sim 2 e^{-\xi} .
\end{aligned}
$$

Next, we calculate the integration over $\mathbb{D}_{r}(1)$. Let $t$ denote the distance from $z$ to $\partial \mathrm{ID}$. According to Lemma 4 and (1), there exist constants $c_{1}$ and $c_{2}$ such that $\nu_{r}(z) \geq$ $\frac{1}{2} \pi r^{2}+c_{1} r t$ and $r \theta(z, r) \leq c_{2} t$. Then,

$$
\begin{aligned}
& \frac{n^{2}}{2} \int_{r=\frac{r_{\xi}}{2}}^{\frac{R_{n}}{2}} \int_{z \in \mathbb{D}_{r}(1)} e^{-n \nu_{r}(z)} 4 r \theta(z, r) d z d r \\
& \leq O(1) n^{2} \int_{r=\frac{r_{\xi}}{2}}^{\frac{R_{n}}{2}} \int_{t=0}^{r} e^{-n\left(\frac{1}{2} \pi r^{2}+c_{1} r t\right)} t d t d r \\
& \leq O(1) n^{2} e^{-\frac{1}{8} n \pi r_{\xi}^{2}} \int_{r=\frac{r_{\xi}}{2}}^{\frac{R_{n}}{2}} \int_{t=0}^{r} e^{-c_{1} n r t} t d t d r \\
& =O(1) n^{2} e^{-\frac{1}{2}(\ln n+\xi)} \int_{r=\frac{r_{\xi}}{2}}^{\frac{R_{n}}{2}} \int_{t=0}^{r} e^{-c_{1} n r t} t d t d r \\
& \leq O(1) n^{2} e^{-\frac{1}{2}(\ln n+\xi)} \int_{r=\frac{r_{\xi}}{2}}^{\frac{R_{n}}{2}} \int_{t=0}^{\infty} e^{-c_{1} n r t} t d t d r \\
& =O(1) n^{2} e^{-\frac{1}{2}(\ln n+\xi)} \int_{r=\frac{r_{\xi}}{2}}^{\frac{R_{n}}{2}}(n r)^{-2} d r \leq O(1) n^{2} e^{-\frac{1}{2}(\ln n+\xi)}\left(n r_{\xi}\right)^{-2} R_{n} \\
& \leq O(1) e^{-\frac{1}{2}(\ln n+\xi)}\left(\sqrt{\frac{\ln n}{n}}\right)^{-1}=O(1)(\ln n)^{-1 / 2}=o(1) .
\end{aligned}
$$

Now, we calculate the integration over $\mathbb{D}_{r}(2)$. Since $\theta(z, r)=0$ for any $z \in \mathbb{D}_{r}(2)$,

$$
\frac{n^{2}}{2} \int_{r=\frac{r_{\xi}}{2}}^{\frac{R_{n}}{2}} \int_{z \in \mathbb{D}_{r}(2)} e^{-n \nu_{r}(z)} 4 r \theta(z, r) d z d r=0 .
$$

Therefore,

$$
\frac{n^{2}}{2} \int_{r=\frac{\xi}{2}}^{\frac{R_{n}}{2}} \int_{z \in \mathbb{D})} e^{-n \nu_{r}(z)} 4 r \theta(z, r) d z d r \sim 2 e^{-\xi} .
$$

Proof of Lemma 12. Let $S$ denote the set of $\left(z_{1}, z_{2}, \cdots, z_{k}\right) \in$ $C_{k 1}\left(\frac{R_{n}}{2}\right)$ satisfying that $z_{1}$ is the one with largest norm among $z_{1}, \cdots, z_{k}$ and $z_{2}$ is the one with longest distance from $z_{1}$ among $z_{2}, \cdots, z_{k}$. Then,

$$
\begin{aligned}
& \left(\frac{n^{2}}{2}\right)^{k} \int_{r \in\left[\frac{\xi}{2}, \frac{R_{n}}{2}\right]^{k}} \int_{z \in C_{k 1}\left(\frac{R_{n}}{2}\right)} \mathbf{1}_{z}^{r} e^{-n \nu_{r}(z)} \prod_{i=1}^{k} 4 r_{i} \theta\left(z_{i}, r_{i}\right) d z_{i} d r_{i} \\
& \leq k(k-1)\left(\frac{n^{2}}{2}\right)^{k} \int_{r \in\left[\frac{\xi}{2}, \frac{R_{n}}{2}\right]^{k}} \int_{z \in S} \mathbf{1}_{z}^{r} e^{-n \nu_{r}(z)} \prod_{i=1}^{k} 4 r_{i} \theta\left(z_{i}, r_{i}\right) d z_{i} d r_{i} .
\end{aligned}
$$

So, it suffices to prove

$$
n^{2 k} \int_{r \in\left[\frac{\xi}{2}, \frac{R_{n}}{2}\right]^{k}} \int_{z \in S} \mathbf{1}_{z}^{r} e^{-n \nu_{r}(z)} \prod_{i=1}^{k} r_{i} \theta\left(z_{i}, r_{i}\right) d z_{i} d r_{i}=o(1) .
$$

Note that, for any $\left(z_{1}, z_{2}, \cdots, z_{k}\right) \in S$, if $\mathbf{1}_{z}^{r}=1$,

$$
\nu_{r_{1}}\left(z_{1}\right)+c R_{n}\left\|z_{2}-z_{1}\right\| \leq \nu_{r}(z) \leq k \frac{1}{4} \pi R_{n}^{2}
$$

for some constant $c$ by Corollary 6 , otherwise, $\mathbf{1}_{z}^{r}=0$, and $z_{i} \in B\left(z_{1},\left\|z_{2}-z_{1}\right\|\right)$, for $3 \leq i \leq k ; \quad z_{2} \in B\left(z_{1},(k-1) R_{n}\right)$.

Thus,

$$
\begin{aligned}
& n^{2 k} \int_{r \in\left[\frac{r_{\xi}}{2}, \frac{R_{n}}{2}\right]^{k}} \int_{z \in S} \mathbf{1}_{z}^{r} e^{-n \nu_{r}(z)} \prod_{i=1}^{k} r_{i} \theta\left(z_{i}, r_{i}\right) d z_{i} d r_{i} \\
& \leq n^{2 k} \int_{r \in\left[\frac{r_{\xi}}{2}, \frac{R n}{2}\right]^{k}} \int_{z \in S} e^{-n\left(\nu_{r_{1}}\left(z_{1}\right)+c R_{n}\left\|z_{2}-z_{1}\right\|\right)} \prod_{i=1}^{k} r_{i} \theta\left(z_{i}, r_{i}\right) d z_{i} d r_{i} \\
& \leq n^{2 k} \int_{r_{1}=\frac{r_{\xi}}{2}}^{\frac{R_{n}}{2}} \int_{\left.z_{1} \in \mathbb{D}\right)} e^{-n \nu_{r_{1}}\left(z_{1}\right)} r_{1} \theta\left(z_{1}, r_{1}\right) d z_{1} d r_{1} \\
& \int_{r_{2}=\frac{\xi}{2}}^{\frac{R_{n}}{2}} 2 \pi r_{2} d r_{2} \int_{z_{2} \in B\left(z_{1},(k-1) R_{n}\right)} e^{-n c R_{n}\left\|z_{2}-z_{1}\right\|} d z_{2} \\
& \prod_{i=3}^{k} \int_{r_{i}=\frac{r_{\xi}}{2}}^{\frac{R_{n}}{2}} 2 \pi r_{i} d r_{i} \int_{z_{i} \in B\left(z_{1},\left\|z_{2}-z_{1}\right\|\right)} d z_{i} \\
& \leq O(1) n^{2 k}\left(R_{n}\left(R_{n}-r_{\xi}\right)\right)^{k-1} \int_{r_{1}=\frac{r_{\xi}}{2}}^{\frac{R_{n}}{2}} \int_{\left.z_{1} \in \mathbb{D}\right)} e^{-n \nu_{r_{1}}\left(z_{1}\right)} r_{1} \theta\left(z_{1}, r_{1}\right) d z_{1} d r_{1} \\
& \int_{z_{2} \in B\left(z_{1},(k-1) R_{n}\right)} e^{-n c R_{n}\left\|z_{2}-z_{1}\right\|}\left\|z_{2}-z_{1}\right\|^{2(k-2)} d z_{2} \\
& \leq O(1) n^{2 k}\left(R_{n}\left(R_{n}-r_{\xi}\right)^{k-1} \int_{r_{1}=\frac{r_{\xi}}{2}}^{\frac{R_{n}}{2}} \int_{\left.z_{1} \in \mathbb{D}\right)} e^{-n \nu_{r_{1}}\left(z_{1}\right)}\right. \\
& r_{1} \theta\left(z_{1}, r_{1}\right) d z_{1} d r_{1} \int_{0}^{\infty} e^{-n c R_{n} \rho} \rho^{2 k-3} d \rho \\
& =O(1) \frac{n^{2 k}\left(R_{n}\left(R_{n}-r_{\xi}\right)\right)^{k-1}}{\left(n R_{n}\right)^{2(k-1)}} \int_{r_{1}=\frac{r_{\xi}}{2}}^{\frac{R_{n}}{2}} \int_{\left.z_{1} \in \mathbb{D}\right)} e^{-n \nu_{r_{1}}\left(z_{1}\right)} \\
& r_{1} \theta\left(z_{1}, r_{1}\right) d z_{1} d r_{1} \\
& =O(1)\left(\frac{R_{n}-r_{\xi}}{R_{n}}\right)^{k-1}\left(2 e^{-\xi}\right)=o(1) \text {. }
\end{aligned}
$$


Here, $\rho=\left\|z_{2}-z_{1}\right\|$, the second to last equality follows from Lemma 11, and the last equality is based on $\frac{R_{n}-r_{\xi}}{R_{n}}=o(1)$.

Proof of Lemma 13. For any $m$-partition $\Pi=\left\{K_{1}, K_{2}\right.$, $\left.\cdots, K_{m}\right\}$ of $\{1,2, \cdots, k\}$, let $\mathbb{D}^{k}(\Pi)$ denote the set of $\left(z_{1}, z_{2}, \cdots, z_{k}\right) \in \mathbb{D}^{k}$ such that, for any $1 \leq j \leq m$, the $\bigcup_{i \in K_{j}} B\left(z_{i}, \frac{R_{n}}{2}\right)$ forms a connected component. Then, $C_{k m}\left(\frac{R_{n}}{2}\right)$ is the union of $\mathbb{D}^{k}(\Pi)$ over all $m$-partitions $\Pi$ of $\{1,2, \cdots, k\}$. So, it is sufficient to show that, for any $m$-partition $\Pi$ of $\{1,2, \cdots, k\}$,

$n^{2 k} \int_{r \in\left[\frac{\xi}{2}, \frac{R_{n}}{2}\right]^{k}} \int_{z \in \mathbb{D}^{k}(\Pi)} \mathbf{1}_{z}^{r} e^{-n \nu_{r}(z)} \prod_{i=1}^{k} r_{i} \theta\left(z_{i}, r_{i}\right) d z_{i} d r_{i}=o(1)$.

Now, fix an $m$-partition $\Pi=\left\{K_{1}, K_{2}, \cdots, K_{m}\right\}$ of $\{1,2, \cdots, k\}$. For $1 \leq j \leq m$, let $l_{j}=\left|K_{j}\right|$, and $r^{(j)}$ and let $z^{(j)}$, respectively, denote the subsequence of $r$ and $z$ corresponding to $K_{j}$. Then,

$$
\mathbb{D}^{k}(\Pi) \subseteq \prod_{j=1}^{m} C_{l_{j} 1}\left(\frac{R_{n}}{2}\right)
$$

and for any $z \in \mathbb{D}^{k}(\Pi)$,

$$
\nu_{r}(z)=\sum_{j=1}^{m} \nu_{r^{(j)}}\left(z^{(j)}\right)
$$

Thus,

$$
\begin{aligned}
& n^{2 k} \int_{r \in\left[\frac{r_{\xi}}{2}, \frac{R_{n}}{2}\right]^{k}} \int_{z \in \mathbb{D}^{k}(\Pi)} \mathbf{1}_{z}^{r} e^{-n \nu_{r}(z)} \prod_{i=1}^{k} r_{i} \theta\left(z_{i}, r_{i}\right) d z_{i} d r_{i} \\
& =n^{2 k} \int_{r \in\left[\frac{r^{\xi}}{2}, \frac{R_{n}}{2}\right]^{k}} \int_{z i n \mathbb{D}^{k}(\Pi)} \mathbf{1}_{z}^{r} e^{-n \sum_{j=1}^{m} \nu_{r^{(j)}}\left(z^{(j)}\right)} \prod_{i=1}^{k} r_{i} \theta\left(z_{i}, r_{i}\right) d z_{i} d r_{i} \\
& =n^{2 k} \int_{r \in\left[\frac{r_{\xi}}{2}, \frac{R_{n}}{2}\right]^{k}} \int_{z \in \mathbb{D}^{k}(\Pi)} \mathbf{1}_{z}^{r} \prod_{j=1}^{m} e^{-n \nu_{r}(j)}\left(z^{(j)}\right) \prod_{i=1}^{k} r_{i} \theta\left(z_{i}, r_{i}\right) d z_{i} d r_{i} \\
& =n^{2 k} \prod_{j=1}^{m} \int_{r \in\left[\frac{r_{\xi}}{2}, \frac{R_{n}}{2}\right]^{l_{j}}} \int_{z \in C_{l^{1}}\left(\frac{R_{n}}{2}\right)} \mathbf{1}_{z}^{r} e^{-n \nu_{r}(z)} \prod_{i=1}^{l_{j}} r_{i} \theta\left(z_{i}, r_{i}\right) d z_{i} d r_{i} \\
& =\prod_{j=1}^{m}\left(n^{2 l_{j}} \int_{r \in\left[\frac{\xi_{k}}{2}, \frac{R_{n}}{2}\right]^{l_{j}}} \int_{z \in C_{l^{1}}\left(\frac{R_{n}}{2}\right)} \mathbf{1}_{z}^{r} e^{-n \nu_{r}(z)} \prod_{i=1}^{l_{j}} r_{i} \theta\left(z_{i}, r_{i}\right) d z_{i} d r_{i}\right) \\
& =o(1) \text {, }
\end{aligned}
$$

where the last equality follows from Lemma 12 and the fact that at least one $l_{j} \geq 2$.

Proof of Lemma 14. For any $z \in C_{k k}\left(\frac{R_{n}}{2}\right)$,

$$
\nu_{r}(z)=\sum_{i=1}^{k} \nu_{r_{i}}\left(z_{i}\right)
$$

Thus,

$$
\begin{aligned}
&\left(\frac{n^{2}}{2}\right)^{k} \int_{r \in\left[\frac{\xi}{2}, \frac{R_{n}}{2}\right]^{k}} \int_{z \in C_{k k}\left(\frac{R_{n}}{2}\right)} e^{-n \nu_{r}(z)} \prod_{i=1}^{k} 4 r_{i} \theta\left(z_{i}, r_{i}\right) d z_{i} d r_{i} \\
&=\left(\frac{n^{2}}{2}\right)^{k} \int_{r \in\left[\frac{\xi}{2}, \frac{R_{n}}{2}\right]^{k}} \int_{z \in C_{k k}\left(\frac{R_{n}}{2}\right)} e^{-n\left(\sum_{i=1}^{k} \nu_{r_{i}}\left(z_{i}\right)\right)} \\
& \\
& \quad \prod_{i=1}^{k} 4 r_{i} \theta\left(z_{i}, r_{i}\right) d z_{i} d r_{i} \\
&=\left(\frac{n^{2}}{2}\right)^{k} \int_{r \in\left[\frac{r_{\xi}}{2}, \frac{R_{n}}{2}\right]^{k}} \int_{z \in \mathbb{D}^{k}} e^{-n\left(\sum_{i=1}^{k} \nu_{r_{i}}\left(z_{i}\right)\right)} \prod_{i=1}^{k} 4 r_{i} \theta\left(z_{i}, r_{i}\right) d z_{i} d r_{i} \\
&-\left(\frac{n^{2}}{2}\right)^{k} \int_{r \in\left[\frac{r_{\xi}}{2}, \frac{R_{n}}{2}\right]^{k}} \int_{z \in \mathbb{D}^{k} \backslash C_{k k}\left(\frac{R_{n}}{2}\right)} e^{-n\left(\sum_{i=1}^{k} \nu_{r_{i}}\left(z_{i}\right)\right)} \\
& \prod_{i=1}^{k} 4 r_{i} \theta\left(z_{i}, r_{i}\right) d z_{i} d r_{i} .
\end{aligned}
$$

We shall show that the first term is asymptotically equal to $\left(2 e^{-\xi}\right)^{k}$, and the second term is asymptotically negligible. Indeed,

$$
\begin{aligned}
& \left(\frac{n^{2}}{2}\right)^{k} \int_{r \in\left[\frac{r_{\xi}}{2}, \frac{R_{n}}{2}\right]^{k}} \int_{z \in \mathbb{D}^{k}} e^{-n\left(\sum_{i=1}^{k} \nu_{r_{i}}\left(z_{i} t\right)\right)} \prod_{i=1}^{k} 4 r_{i} \theta\left(z_{i}, r_{i}\right) d z_{i} d r_{i} \\
& =\left(\frac{n^{2}}{2}\right)^{k} \int_{r \in\left[\frac{r_{\xi}}{2}, \frac{R_{n}}{2}\right]^{k}} \int_{z \in \mathbb{D}} \prod_{i=1}^{k} e^{-n \nu_{r_{i}}\left(z_{i}\right)} 4 r_{i} \theta\left(z_{i}, r_{i}\right) d z_{i} d r_{i} \\
& =\prod_{i=1}^{k}\left(\frac{n^{2}}{2} \int_{r=\frac{r_{\xi}}{2}}^{\frac{R_{n}}{2}} \int_{z \in \mathbb{D}} e^{-n \nu_{r}(z)} 4 r \theta(z, r) d z d r\right) \sim\left(2 e^{-\xi}\right)^{k},
\end{aligned}
$$

where the last equality follows from Lemma 11. If $\left(z_{1}, z_{2}, \cdots, z_{k}\right) \in C_{k 1}\left(\frac{R_{n}}{2}\right)$ satisfying that $z_{1}$ is the one with largest norm among $z_{1}, \cdots, z_{k}$ and $z_{2}$ is the one with longest distance from $z_{1}$ among $z_{2}, \cdots, z_{k}$, it can be proved that

$$
\nu_{r_{1}}\left(z_{1}+c R_{n}\left\|z_{1}-z_{2}\right\| \leq \sum_{i=1}^{k} \nu_{r_{i}}\left(z_{i}\right) \leq k \frac{1}{4} \pi R_{n}^{2} .\right.
$$

Note that this is similar to Corollary 6, but with no need of the feasible condition. Thus,

$$
\begin{aligned}
& \left(\frac{n^{2}}{2}\right)^{k} \int_{r \in\left[\frac{r_{k}}{2}, \frac{R_{n}}{2}\right]^{k}} \int_{z \in \mathbb{D}^{k} \backslash C_{k k}\left(\frac{R_{n}}{2}\right)} e^{-n \sum_{i=1}^{k} \nu_{r_{i}}\left(z_{i}\right)} \prod_{i=1}^{k} 4 r_{i} \theta\left(z_{i}, r_{i}\right) d z_{i} d r_{i} \\
& \leq \sum_{m=1}^{k-1}\left(\frac{n^{2}}{2}\right)^{k} \int_{r \in\left[\frac{\xi}{2}, \frac{R_{n}}{2}\right]^{k}} \int_{z \in C_{k m}\left(\frac{R_{n}}{2}\right)} e^{-n \sum_{i=1}^{k} \nu_{r_{i}}\left(z_{i}\right)} \prod_{i=1}^{k} 4 r_{i} \theta\left(z_{i}, r_{i}\right) d z_{i} d r_{i} \\
& =o(1),
\end{aligned}
$$

where the last equality can be proved by the arguments used in Lemma 12 and Lemma 13.

Proof of Lemma 15. Without loss of generality, assume $i=1$. In addition, assume $j=2$. (If $1 \leq j \leq t$, the following proof still works. If $t+1 \leq j \leq m-t$, the following proof works after some minor modifications.) Note that

$$
\begin{aligned}
& z_{i} \in B\left(z_{1},\left\|z_{2}-z_{1}\right\|\right), \text { for } 3 \leq i \leq m-t \\
& z_{2} \in B\left(z_{1},(m-t-1) R_{n}\right) .
\end{aligned}
$$


(In the following, we assume $t \geq 2$.) Thus,

$$
\begin{aligned}
& n^{m} \int_{r \in\left[\frac{\xi}{2}, \frac{R_{n}}{2}\right]^{t}} \int_{z \in S^{i j}} e^{-n\left(\nu_{r_{i}}\left(z_{i}\right)+c R_{n}\left\|z_{i}-z_{j}\right\|\right)}\left(\prod_{k=1}^{t} r_{k} \theta\left(z_{k}, r_{k}\right) d r_{k}\right) \\
& \left(\prod_{k=1}^{m-t} d z_{k}\right) \\
& \leq O(1) n^{m}\left(\int_{r_{1}=\frac{r_{\xi}}{2}}^{\frac{R_{n}}{2}} \int_{\left.z_{1} \in \mathbb{D}\right)} e^{-n \nu_{r_{1}}\left(z_{1}\right)} r_{1} \theta\left(z_{1}, r_{1}\right) d z_{1} d r_{1}\right) \\
& \left(\int_{z_{2} \in B\left(z_{1},(m-t-1) R_{n}\right)} e^{-n c R_{n}\left\|z_{2}-z_{1}\right\|} d z_{2}\right) \\
& \left(\prod_{i=2}^{t} \int_{r_{i}=\frac{r_{\xi}}{2}}^{\frac{R_{n}}{2}} \pi r_{i} d r_{i}\right)\left(\prod_{i=3}^{m-t} \int_{z_{i} \in B\left(z_{1},\left\|z_{2}-z_{1}\right\|\right)} d z_{i}\right) \\
& \leq O(1) n^{m}\left(R_{n}\left(R_{n}-r_{\xi}\right)\right)^{t-1} \int_{r_{1}=\frac{r_{\xi}}{2}}^{\frac{R_{n}}{2}} \int_{\left.z_{1} \in \mathbb{D}\right)} e^{-n \nu_{r_{1}}\left(z_{1}\right)} \\
& r_{1} \theta\left(z_{1}, r_{1}\right) d z_{1} d r_{1} \\
& \int_{z_{2} \in B\left(z_{1},(k-1) R_{n}\right)} e^{-n c R_{n}\left\|z_{2}-z_{1}\right\|}\left\|z_{2}-z_{1}\right\|^{2(m-t-2)} d z_{2} \\
& \leq O(1) n^{m}\left(R_{n}\left(R_{n}-r_{\xi}\right)\right)^{t-1} \int_{r_{1}=\frac{r_{\xi}}{2}}^{\frac{R_{n}}{2}} \int_{\left.z_{1} \in \mathbb{D}\right)} e^{-n \nu_{r_{1}}\left(z_{1}\right)} \\
& r_{1} \theta\left(z_{1}, r_{1}\right) d z_{1} d r_{1} \int_{0}^{\infty} e^{-n c R_{n} \rho} \rho^{2(m-t)-3} d \rho \\
& =O(1) \frac{n^{m}\left(R_{n}\left(R_{n}-r_{\xi}\right)\right)^{t-1}}{\left(n R_{n}\right)^{2(m-t-1)}} \int_{\left[r_{\xi}, R_{3}\right]} \int_{\left.z_{1} \in \mathbb{D}\right)} e^{-n \nu_{r_{1}}\left(z_{1}\right)} \\
& r_{1} \theta\left(z_{1}, r_{1}\right) d z_{1} d r_{1} \\
& =O(1) \frac{n^{m-2}\left(\frac{R_{n}\left(\xi_{n}-\xi\right)}{\left(R_{n}-r_{\xi}\right) \pi n}\right)^{t-1}}{n^{m-t-1}\left(n R_{n}^{2}\right)^{m-t-1}} \\
& \left(n^{2} \int_{r_{1}=\frac{r_{\xi}}{2}}^{\frac{R_{n}}{2}} \int_{\left.z_{1} \in \mathbb{D}\right)} e^{-n \nu_{r_{1}}\left(z_{1}\right)} r_{1} \theta\left(z_{1}, r_{1}\right) d z_{1} d r_{1}\right) \\
& =O(1)\left(\frac{\left(\xi_{n}-\xi\right)^{t-1}}{\ln ^{m-t-1} n}\right)\left(e^{-\xi}\right)=o(1),
\end{aligned}
$$

where the second to last equality follows from Lemma 11 and the last equality is based on $\xi_{n}=o(\ln n)$ and $m-t-1 \geq 1$.

\section{ACKNOWLEDGMENTS}

The work of P.-J. Wang is supported in part by the US National Science Foundation (NSF) under Grant 557904 and CityU of Hong Kong under Grant 7200031. The work of C.W. Yi was supported in part by the NSC under Grant NSC94-2218-E-009-030 and NSC95-2221-E-009-059-MY3, by MOE ATU Program, and by Intel JRP, and was partially done when the author visited the CityU of Hong Kong.

\section{REFERENCES}

[1] N. Li, J.C. Hou, and L. Sha, "Design and Analysis of a MST-Based Distributed Topology Control Algorithm for Wireless Ad-Hoc Networks," Proc. 22nd Ann. Joint Conf. IEEE Computer and Comm. Soc. (INFOCOM '03), vol. 3, pp. 1702-1712, Apr. 2003.
[2] Y. Wang and X.-Y. Li, "Localized Construction of Bounded Degree and Planar Spanner for Wireless Ad Hoc Networks," Proc. 2003 Joint Workshop Foundations of Mobile Computing (DIALM-POMC '03), pp. 59-68, Sept. 2003.

[3] J. Cartigny, F. Ingelrest, D. Simplot-Ryl, and I. Stojmenovic, "Localized LMST and RNG Based Minimum-Energy Broadcast Protocols in Ad Hoc Networks," Ad Hoc Networks, vol. 3, no. 1, pp. 1-16, 2004.

[4] E.N. Gilbert, "Random Plane Networks," J. Soc. for Industrial and Applied Math., vol. 9, no. 4, pp. 533-543, Dec. 1961.

[5] P. Gupta and P.R. Kumar, "Critical Power for Asymptotic Connectivity in Wireless Networks," Stochastic Analysis, Control, Optimization and Applications: A Volume in Honor of W.H. Fleming, W.M. McEneaney, G. Yin, and Q. Zhang, eds., pp. 547-566, Birkhauser, Mar. 1998.

[6] H. Dette and N. Henze, "The Limit Distribution of the Largest Nearest-Neighbour Link in the Unit $d$-Cube," J. Applied Probability, vol. 26, pp. 67-80, 1989.

[7] M.D. Penrose, "The Longest Edge of the Random Minimal Spanning Tree," The Annals of Applied Probability, vol. 7, no. 2, pp. 340-361, 1997.

[8] G. Kozma, Z. Lotker, M. Sharir, and G. Stupp, "Geometrically Aware Communication in Random Wireless Networks," Proc. 23rd Ann. ACM Symp. Principles of Distributed Computing, pp. 310319, July 2004.

[9] P.-J. Wan and C.-W. Yi, "Asymptotic Critical Transmission Ranges for Connectivity in Wireless Ad Hoc Networks With Bernoulli Nodes," Proc. IEEE Wireless Comm. and Networking Conf. (WCNC '05), Mar. 2005.

[10] C.-W. Yi, P.-J. Wan, X.-Y. Li, and O. Frieder, "Asymptotic Distribution of the Number of Isolated Nodes in Wireless Ad Hoc Networks with Bernoulli Nodes," Proc. IEEE Wireless Comm. and Networking Conf. (WCNC '03), Mar. 2003.

[11] M. Penrose, Random Geometric Graphs. Oxford Univ. Press, 2003.

[12] H. Zhang and J.C. Hou, "On the Critical Total Power for Asymptotic $k$-Connectivity in Wireless Networks," Proc. 24th Ann. Joint Conf. IEEE Computer and Comm. Soc. (INFOCOM '05), Mar. 2005.

[13] N. Alon and J.H. Spencer, The Probabilistic Method, second ed. Wiley, Mar. 2000.

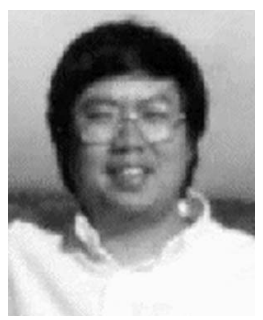

Peng-Jun Wan received the PhD degree from the University of Minnesota, the MS degree from The Chinese Academy of Science, and the BS degree from Tsinghua University. He is currently an associate professor of computer science at the Illinois Institute of Technology and the City University of Hong Kong. His research interests include wireless networks, optical networks, and algorithm design and analysis.

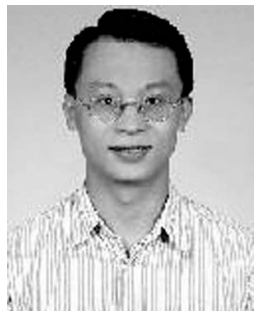

Chih-Wei Yi received the PhD degree from the Illinois Institute of Technology, and the MS and BS degrees from National Taiwan University. He is currently an assistant professor in the Department of Computer Science, National Chiao Tung University. His research focuses on wireless ad hoc networks. He is a member of the IEEE.

$\triangleright$ For more information on this or any other computing topic, please visit our Digital Library at www.computer.org/publications/dlib. 